\title{
Optimization of Visible-Light Optical Wireless Systems: Network-Centric Versus User-Centric Designs
}

\author{
Xuan Li, Rong Zhang, Lajos Hanzo
}

\begin{abstract}
In order to counteract the explosive escalation of wireless tele-traffic, the communication spectrum has been gradually expanded from the conventional radio frequency (RF) band to the optical wireless $(\mathrm{OW})$ domain. By integrating the classic RF band relying on diverse radio techniques and optical bands, the next-generation heterogeneous networks (HetNets) are expected to offer a potential solution for supporting the ever-increasing wireless tele-traffic. Owing to its abundant unlicensed spectral resources, visible light communications (VLC) combined with advanced illumination constitute a competent candidate for complementing the existing RF networks. Although the advantages of VLC are multi-fold, some challenges arise when incorporating VLC into the classic RF HetNet environments, which may require new system architectures. The user-centric (UC) design principle for VLC environments constitutes a novel and competitive design paradigm for the super dense multi-tier cell combinations of HetNets. The UC concept may be expected to become one of the disruptive techniques to be used in the forthcoming fifthgeneration era. This paper provides a comprehensive survey of visible-light-aided $O W$ systems with special emphasis on the design and optimization of VLC networks, where the radically new UC design philosophy is reviewed. Finally, design guidelines are provided for VLC systems.
\end{abstract}

\section{INTRODUCTION}

As a truly revolutionary paradigm shift [1], [2], wireless technology relying on radio waves has become an integral part of our everyday life, facilitating basic services, such as making a phone call or sending a message, as well as nearfield-communication-aided mobile payment. The radio waves occupy the $3 \mathrm{kHz} 300 \mathrm{GHz}$ electromagnetic band, which has been widely used for communication purposes. However, with the increase of tele-traffic, as recently predicted by Cisco and shown in Figure 1, the radio frequency (RF) band is becoming more and more crowded. Following the launch of the global fifth generation (5G) research initiatives [3] conceived for tackling the explosive escalation of wireless tele-traffic, the horizon of communication bands has been expanded from the conventional RF band both to the millimeter wave [4] and to the visible light [1] frequency band spanning from 400 to 800 THz.

The earliest known use of visible light communications (VLC) dates back to 1880, when Bell developed a photophone

The financial support of the European Research Council's Advanced Fellow Grant Beam-Me-Up, of the Chinese Scholarship Council as well as of the EPSRC UK under the contracts EP/N004558/1 and EP/L018659/1 is gratefully acknowledged.

\begin{tabular}{|ll|}
\hline & \multicolumn{1}{c|}{ ABBREVIATIONS } \\
\hline 5G & Fifth generation \\
ACO-OFDM & Asymmetrically clipped optical OFDM \\
AP & Access point \\
ASE & Area spectral efficiency \\
BER & Bit error rate \\
CIR & Channel's impulse response \\
CSK & Colour shift keying \\
CT & Combined transmission \\
DC & Direct current \\
DCO-OFDM & DC biased optical OFDM \\
DL & Down-link \\
DMT & Discrete multitone \\
FoV & Field-of-view \\
FR & Frequency reuse \\
GCM & Generalized colour modulation \\
GPS & Global positioning system \\
HetNets & Heterogeneous networks \\
ICI & Inter-cell interference \\
IM-DD & Intensity modulation direct detection \\
IoT & Internet-of-Things \\
LB & Load balancing \\
LED & Light-emitting diode \\
Li-Fi & Light fidelity \\
LoS & Line-of-sight \\
MC-LED & Multi-chip LED \\
MIMO & Multiple input multiple output \\
MU-MIMO & Multiuser MIMO \\
MU-MISO & Multiuser-multiple-input-single-output \\
MUS & Multiuser scheduling \\
NLoS & Non-LoS \\
O/E & Optical/electronic \\
OLED & Organic LED \\
OOFDM & Optical orthogonal frequency division multiplexing \\
OOK & On-off keying \\
OW & Optical wireless \\
PAM & Pulse amplitude modulation \\
PC-LED & Phosphor converted LED \\
PD & Photodetector \\
PDP & power delay profile \\
PLC & power-line communication \\
PPM & Pulse position modulation \\
QAM & Quadrature amplitude modulation \\
QoS & Quality of service \\
RF & Radio frequency \\
SHVC & Scalable extension of the high-efficiency video coding \\
SINR & Signal-to-interference-plus-noise ratio \\
SISO & Single input single output \\
SNR & Signal-to-noise ratio \\
U-OFDM & Unipolar OFDM \\
UC & User-centric \\
UC-VT & User-centric-vectored-transmission-aided \\
UE & User equipment \\
UFR & Unity FR \\
V2I & Vehicle to infrastructure \\
V2V & Vehicle to vehicle \\
VHO & Vertical handover \\
VLC & Visible light communications \\
VT & Vectored transmission \\
WiFi & Wireless fidelity \\
\hline
\end{tabular}




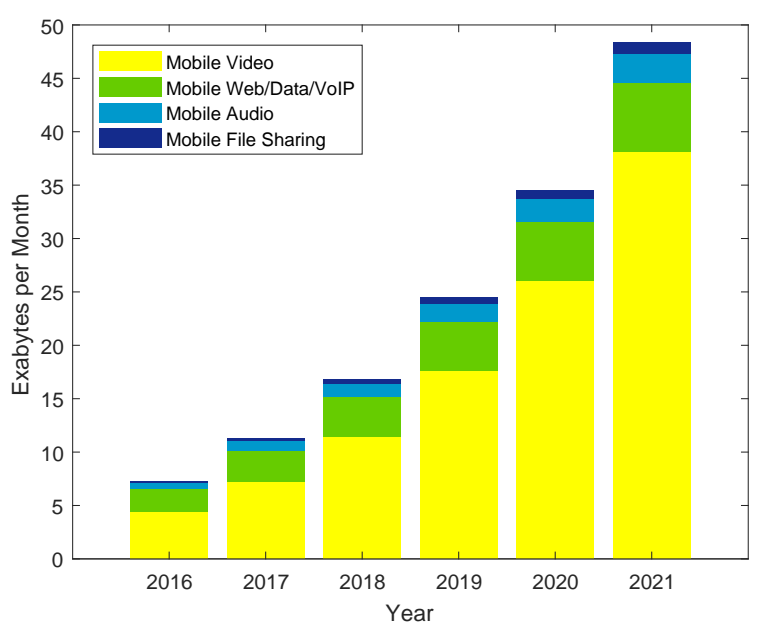

Fig. 1: The global mobile data traffic forecast for 2016-2021 [7].

\begin{tabular}{|l|l|l|}
\hline Property & \multicolumn{1}{|c|}{ VLC } & \multicolumn{1}{c|}{ RF } \\
\hline Services & $\begin{array}{l}\text { Illumination and } \\
\text { communications }\end{array}$ & Communications \\
\hline Bandwidth & $\begin{array}{l}\text { Unregulated, 400 } \\
\mathrm{THz}-800 \mathrm{THz}\end{array}$ & $\begin{array}{l}\text { Typically regu- } \\
\text { lated, up to 300 } \\
\mathrm{GHz}\end{array}$ \\
\hline Coverage & Limited & Wide \\
\hline Distance & Short & Short to long \\
\hline Security & Good & Poor \\
\hline Mobility & Limited & Good \\
\hline
\end{tabular}

TABLE I: Comparison of VLC and RF communications, reproduced from [8].

transmitting voice by employing sunlight [5]. With the development of solid-state lighting, white light-emitting diodes (LEDs) were invoked for constructing VLC systems in the early 2000s for the dual function of illumination and communications by Tanaka et al. in Japan [6]. In VLC, the intensity of light is modulated and transmitted by a light source, which flickers above the fusion-frequency of the human eye. As a benefit of their high switching rate and energy efficiency, LEDs may be the best choice for indoor VLC systems, which are capable of serving the dual function of illumination and communications.

The specific features of VLC contrasted to traditional RF communications are in Table I. Relying on the unlicensed spectrum spanning from $400 \mathrm{THz}-800 \mathrm{THz}$, VLC is expected to be an integral part of next-generation communication systems with the dual function of illumination and communications. Due to the line-of-sight proporgation of light, each VLC access point can only illuminate a small cell, but cannot propagate through walls. Hence, it is more secure than RF communications, but supportive of less mobility.

\section{A. Related Works}

The research of VLC has been intensified during the past decade or so. Valuable efforts have been invested in surveying this field [9]-[20]. To elaborate, a brief survey [9] of VLC systems was published in 2010, which discussed the VLC techniques and their applications in their early development phase. Tsonev et al. [10] discussed some of the key networking techniques required for realizing light fidelity (Li-Fi) networks, including the associated multiple access techniques, the potential uplink solutions as well as the optical attocell and overall network structure. Sevincer et al. [11] introduced the concept of the so-called "LIGHTNETs", which represented the dual function of smart lighting and optical wireless (OW) communications, with special emphasis on the inherent design tradeoffs between these two functions [11].

Furthermore, VLC techniques were surveyed as a potential member of the $5 \mathrm{G}$ communication systems by $\mathrm{Wu}$ et al. [12], including both indoor and outdoor communications. Recently, Karunatilaka et al. [13] reviewed the evolutionary development of VLC-based techniques and the related challenges. In another comprehensive survey of the existing literature of VLC systems [14], Pathak et al. discussed the associated link-layer techniques as well as the optical cell design and interferencecoordination schemes, with an additional emphasis on the pertinent issues of visible light sensing.

There are also several survey papers on specific indepth aspects of VLC systems [15]-[20]. For example, Saha et al. [15] reviewed the key techniques in optical camera communication systems by considering the IEEE standard. Qiu et al. [16] reviewed the state-of-the-art in VLC channel modelling methods, in order to provide a better insight into the theoretical basis of VLC system design, while the optical background noise sources and the corresponding noise reduction techniques of VLC systems were investigated by Sindhubala and Vijayalakshmi [18]. The applications of VLC are expected to be extended to the Internet-of-Things (IoT). Explicitly, Do and Yoo [19] provided an in-depth survey of VLC-based positioning systems, while Cailean and Dimian [17] summarized the existing solutions designed for integrating VLC into vehicular communications. Furthermore, Rajbhandari et al. [20] provideds an overview of the recent developments of high-speed gallium-nitride-LED-aided VLC systems. The main contributions of the existing VLC survey papers are contrasted in Table II.

\section{B. Contributions and Organization}

Figure 2 provides a glimpse of the diverse system components and the associated enabling techniques designed for wireless systems, where the general VLC link structure and the unique characteristics of VLC systems are highlighted. To elaborate a little further, for a general wireless system, the ambitious design objectives of maintaining a high throughput, high quality of service (QoS), etc. have to be achieved in the face of the limited resources, including the achievable bandwidth, the implementation complexity, etc. In order to optimize this multi-objective problem, a vast number of valuable wireless enabling techniques have been investigated, as seen in Figure 2. The associated PHY- and network-related techniques are listed in the left and right boxes of Figure 2, respectively. Although the design of a VLC system is to a degree similar to that of a general wireless system, there are also some unique characteristics to be considered. For example, a VLC 


\begin{tabular}{|l|l|l|}
\hline Year & \multicolumn{1}{|c|}{ Paper } & \multicolumn{1}{c|}{ Focus/Main contribution } \\
\hline 2010 & Kumar and Lourenco [9] & VLC techniques and applications in their early development phase \\
\hline 2013 & Sevincer et al. [11] & The inherenet design tradeoffs between lighting and communications \\
\hline 2013 & Tsonev et al. $[10]$ & Key networking techniques in Li-Fi networks \\
\hline 2014 & Wu et al. $[12]$ & VLC as a potential member in 5G communication systems \\
\hline 2015 & Karunatilaka et al. [13] & The evolutionary development of VLC-based techniques and related challenges \\
\hline 2015 & Saha et al. [15] & Key techniques in the optical camera communication systems \\
\hline 2015 & Pathak et al. $[14]$ & Comprehensive survey on link-layer and cell design techniques as well as visible light sensing \\
\hline 2016 & Qiu et al. $[16]$ & VLC channel modelling methods \\
\hline 2016 & Cailean and Dimian et al. $[21]$ & Environmental adaptive VLC receiver for vehicular communication applications \\
\hline 2016 & Sindhubala and Vijayalakshmi [18] & The optical background noise sources and its reduction techniques \\
\hline 2016 & Do and Yoo [19] & VLC-based positioning system \\
\hline 2017 & Rajbhandari et al. [20] & Developments of high-speed gallium-nitride-LED-aided VLC systems \\
\hline \multicolumn{2}{|c|}{ This paper } & System-level VLC networks, from network-centric to user-centric design principle \\
\hline
\end{tabular}

TABLE II: Comparison of different VLC survey papers.

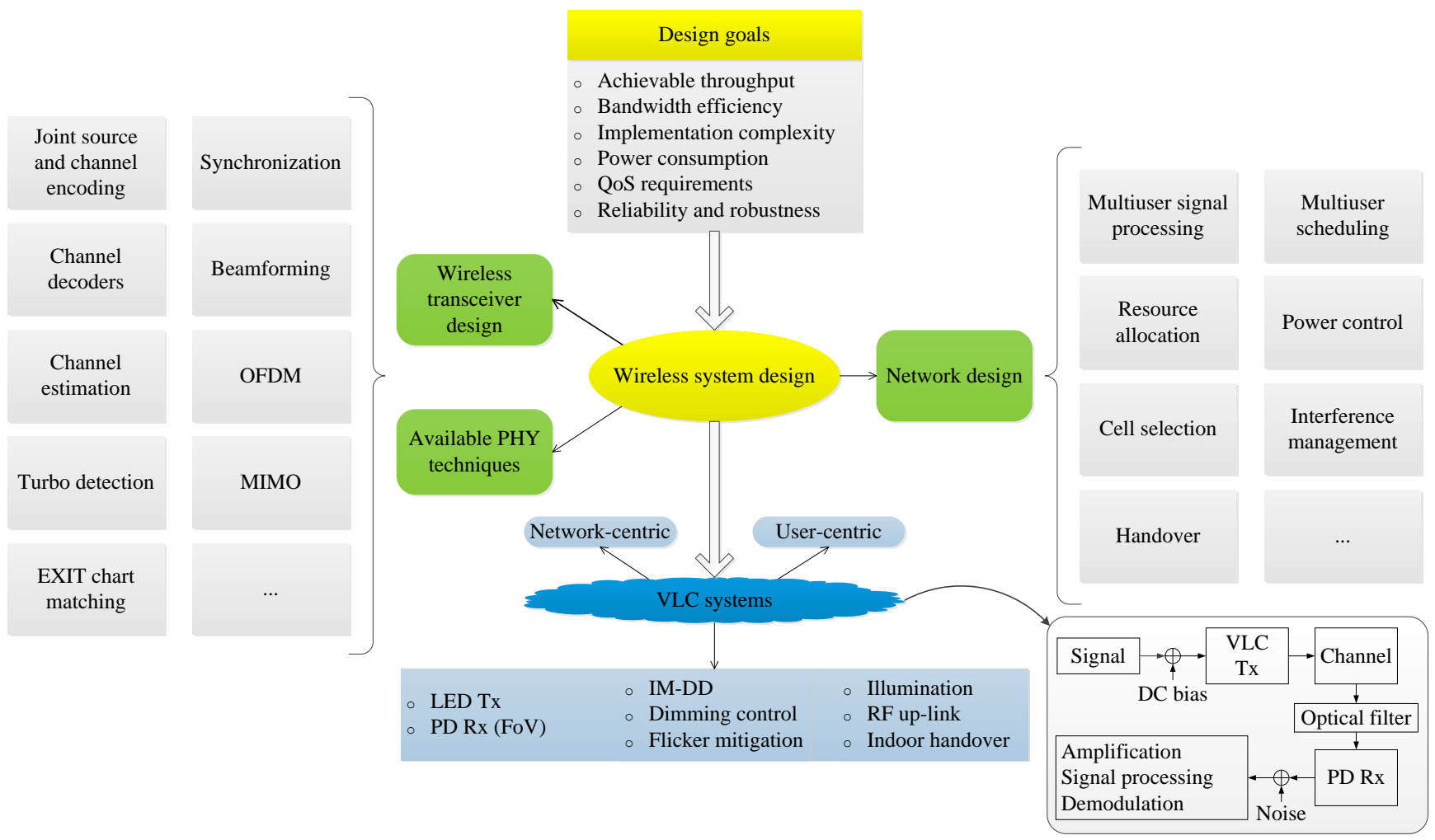

Fig. 2: Diverse system components and factors affecting the design of wireless systems, where the general VLC link structure and the unique characteristics of VLC systems are highlighted.

system generally employs a light-emitting diode (LED)-aided transmitter (Tx) and the photodetector (PD) based receiver $(\mathrm{Rx})$, where the intensity modulated light signals are transmitted. Hence, both the general wireless transceiver design and the modulation techniques have to be appropriately adjusted for VLC systems. Considering the specific characteristics of VLC cells relying on line-of-sight light propagation, the VLC network may require a radically new structure, relying on a user-centric (UC) VLC network [22], where the LEDtransmitters 'follow the users', rather than the other way round. Furthermore, according to the existing literature, the standalone VLC networks may not be capable of providing a convenient uplink. Therefore, the classic RF network can be invoked as a cooperative partner of VLC.
In this paper, an extensive review of the family of VLC techniques invented during the past decades is presented, where our particular attention is focused on the system-level study of the OW networks. Explicitly,

- the link-level characteristics of VLC systems are reviewed from the perspective of point-to-point transmission, including its transceivers, the optical channels as well as the PHY techniques;

- we survey the layout of VLC-aided networks, with special emphasis on interference mitigation techniques specifically designed for VLC-based systems. This issue was also reviewed by Pathak et al. [14]. However, our new contribution is that apart from the conventional networkcentric methods presented in [14], we also survey the 
advanced user-centric cell formation design philosophy;

- the solutions of the salient multiuser signal processing, scheduling and resource management problems in multiuser VLC-aided networks are surveyed;

- the applications and extensions of VLC systems are surveyed, followed by the introduction of the open challenges encountered when integrating VLC into nextgeneration communication systems.

VLC can be considered as a new member in the smallcell family of the heterogeneous networks (HetNets) landscape for complementing the overloaded radio frequency band [22]. The UC cluster formation principle designed for VLC environments constitutes a novel and competitive design paradigm for the super dense multi-tier cell combinations of HetNets, where the sophisticated user equipment (UE) can actively participate in cell planning, resource management, mobility control, service provision, signal processing, etc. As a result, the UC concept may be expected to become one of the disruptive techniques to be used in the forthcoming $5 \mathrm{G}$ era [22].

By definition, similar to the traditional radio frequency cell, the network-centric cell relies on an optical AP, which provides network coverage for a fix location. Multiple neighbouring cells may use different frequencies or cooperate with each other. By contrast, the UC cluster formation design takes the user-distribution into consideration and a typical UC cluster includes the serving VLC APs, the users as well as the relevant optical links. Hence, the network-centric cell is often preset as a fixed shape according to the transmission schemes used, while the UC cluster is amorphous and constructed dynamically by considering the tele-traffic during the current time slot. Furthermore, the network-centric cell design is subject to the specific arrangements of VLC APs, but the UC design is readily adjusted to any AP layout.

Normally, the network-centric cell configuration is fixed and the network resources are allocated to the users according to pre-set algorithms. By contrast, the UC cluster formation and its resource allocation are combined and act in unison. Explicitly, during the cluster formation process, the users are scheduled and the resources are allocated at the same time. Additionally, the intra-cluster interference of the UC cluster formation is eliminated and the inter-cluster interference may be minimized by carefully designing the UC cluster radius. Moreover, the handover mechanism of network-centric networks is triggered by the users, since generally the users send their handover requests, when moving. In UC networks, the handover may be seen as being triggered by APs, where the clusters are dynamically updated by tracking the users. The major differences of network-centric and UC design are summarized in Table III.

In the rest of this paper, point-to-point VLC links will be introduced in Section II including the devices and other components, the VLC channels as well as the physical layer techniques. An overview of VLC networks will be presented in Section III. Furthermore, the applications and the open challenges of VLC-based systems will be discussed in Section IV and Section V, respectively.

\begin{tabular}{|l|l|l|}
\hline Property & Network-centric & User-centric \\
\hline Definition & $\begin{array}{l}\text { AP and its cover- } \\
\text { age }\end{array}$ & AP, users, links \\
\hline Cell shape & Fixed & Amorphous \\
\hline Tele-traffic & Neglect & Consider \\
\hline Adjustability & $\begin{array}{l}\text { Subject to APs ar- } \\
\text { rangements }\end{array}$ & $\begin{array}{l}\text { Adjustable to any } \\
\text { APs arrangements }\end{array}$ \\
\hline $\begin{array}{l}\text { Resource allo- } \\
\text { cation }\end{array}$ & $\begin{array}{l}\text { Separate from cell } \\
\text { formation }\end{array}$ & $\begin{array}{l}\text { Combined with } \\
\text { cluster formation }\end{array}$ \\
\hline Interference & Relatively high & Relatively low \\
\hline Handover & User-triggered & AP-triggered \\
\hline
\end{tabular}

TABLE III: Comparison of network-centric and user-centric design philosophy.

\section{VLC LINKS}

A general VLC link structure is shown in Figure 2. The optically modulated signal is superimposed on the average direct current (DC) level, which drives the LED and converts the electrical signals into intensity modulated optical signals. The received optical signal arrives at the optical filter and it is focused on a photodetector (PD), where the optical signal is converted back to electrical signal. Due to the thermal agitation of charge carriers and owing to the ambient light, noise is imposed. In Figure 2, only the electronic domain noise is shown, although noise is accumulated at every stage. Relying on the process of amplification, signal processing and demodulation, etc. the transmitted signals are then recovered. In this section, VLC is treated as a point-to-point data transmission technique. Its transmitter and receiver, its channel model as well as the applicable modulation techniques will be introduced. Additionally, since the primary function of light luminaries is to provide illumination in indoor environments, adjustable dimming and safety to the human eye should also be considered as constraints, when integrating VLC with indoor lighting systems.

\section{A. Device and Components}

1) VLC Transmitter: Light-Emitting Diode: Owing to the rapid development of solid-state lighting, which refers to the fact that light is emitted by solid-state electro-luminescence as opposed to incandescent bulbs or fluorescent tubes, VLC using LEDs as the data 'transmitters' has intensified during the past decade or so. Exhibiting the dual function of illumination and data transmission, the advantages of LEDs are multi-fold, such as low heat generation [23], low power consumption [24], high energy conversion efficiency/luminous efficacy [25] as well as a potentially high bandwidth [26], etc. Diverse types of LEDs have been developed, which are available in commercial markets, including phosphor converted LEDs (PC-LEDs) [27], multi-chip LEDs (MC-LEDs) [28], organic LEDs (OLEDs) [29] and micro-LEDs [30].

Apart from the study of the specific types of LEDs mentioned above, the basic characteristics of white LEDs have been widely investigated in [13], [14], [24], [26], [31], for the sake of constructing a VLC system. Since LEDs perform dual functions in indoor environments, the typical characteristics of LEDs are usually studied from two perspectives, namely in terms of their photometric parameters and radiometric 
parameters. The photometric parameters quantify the LEDs characteristics in terms of indoor illumination, while the radiometric parameters describe the radiated electromagnetic energy of the light, which are related to the communication characteristics of LEDs. Let us now provide a brief description of the most important parameters:

- luminosity function $V(\lambda)$ : the function reflecting the human eyes' sensitivity to different colours, which provides design guidelines for the associated lighting technology [32];

- spectral power distribution $S_{p}(\lambda)[\mathrm{W} / \mathrm{nm}]$ : the function reflecting the power of the LED at all wavelengths in the visible light spectrum [33];

- luminous flux $\Phi$ [lumen]: calculating the power emitted by an LED, which can be perceived by the human eye [34];

- luminous intensity $I_{\mathcal{L}}$ [candelas]: a parameter defined as the luminous flux per unit solid angle, which describes the brightness of an LED;

- semi-angle at half power: the angle at which the luminous intensity in candelas decreases to half of the intensity at $0^{\circ}$ solid angle, etc.

To elaborate further, the luminous flux $\Phi$ may be characterized as

$$
\Phi=V_{m} \int_{\lambda_{1}}^{\lambda_{2}} V(\lambda) S_{p}(\lambda) \mathrm{d} \lambda,
$$

where $V_{m}$ is the maximum visibility, which is around 683 $\mathrm{lm} / \mathrm{W}$ at $\lambda=555 \mathrm{~nm}$. Furthermore, according to its definition, the luminous intensity $I_{\mathcal{L}}$ may be written as $I_{\mathcal{L}}=\mathrm{d} \Phi / \mathrm{d} \theta$, where $\theta$ is the spatial angle. In order to calculate the illuminance of an illuminated surface, from a different perspective, we may also calculate $I_{\mathcal{L}}$ in angle $\phi$ as

$$
I_{\mathcal{L}}(\phi)=I_{\mathcal{L}}(0) \cdot \cos ^{w}(\phi),
$$

where $I_{\mathcal{L}}(0)$ is the centre luminous intensity of an LED and $\phi$ is the angle of irradiance. Furthermore, $w$ denotes the order of Lambertian emission ${ }^{1}$ and is given by $w=\ln 2 / \ln \left(\cos \phi_{1 / 2}\right)$, where $\phi_{1 / 2}$ is the semi-angle at half-illuminance of the LED. Now we are ready to calculate the illuminance $E_{\mathcal{L}}$ at a certain point of the illuminated surface, which is given as

$$
E_{\mathcal{L}}=I_{\mathcal{L}}(0) \cdot \cos ^{w}(\phi) / d_{a, u}^{2} \cdot \cos (\psi),
$$

where $d_{a, u}$ is the distance between the LED transmitter and the receiver's surface, while $\psi$ is the angle of incidence.

Since illumination is the primary function of LEDs, the illumination requirements should be considered before invoking their communication function. As an example, we investigate the illumination distribution of a typical $15 \mathrm{~m} \times$ $15 \mathrm{~m}$ room covered by $8 \times 8$ uniformly distributed LED-array transmitters at a height of $2.5 \mathrm{~m}$. Our simulation parameters are summarized in Table IV. The $15 \mathrm{~m} \times 15 \mathrm{~m}$ indoor area considered is divided into $100 \times 100$ tiles and the center illumination of each grid can be calculated by substituting the

\footnotetext{
${ }^{1}$ In Lambertian emission, the optical power radiated from a unit area into a unit solid angle is constant [8]. The maximum luminous intensity $I_{\mathcal{L}}(0)$ is perpendicular to the planar surface but reduced proportional by the cosine of the angle $\phi$.
}

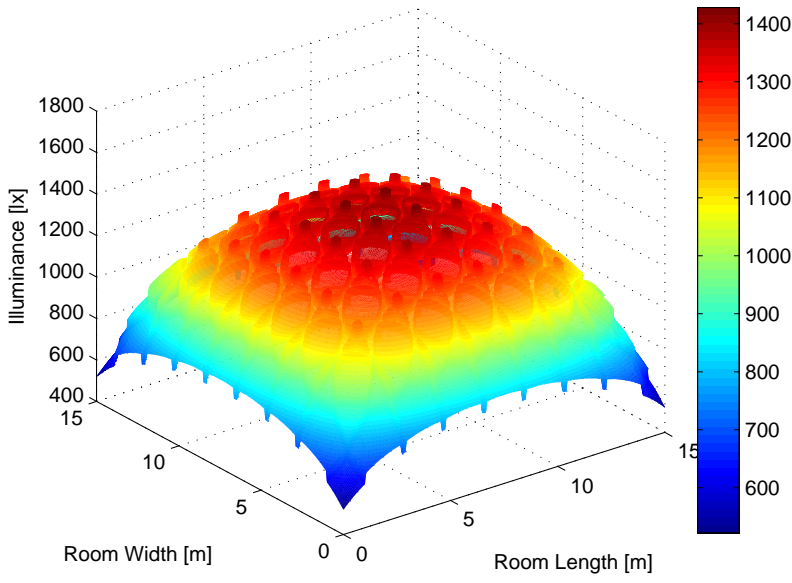

(a)

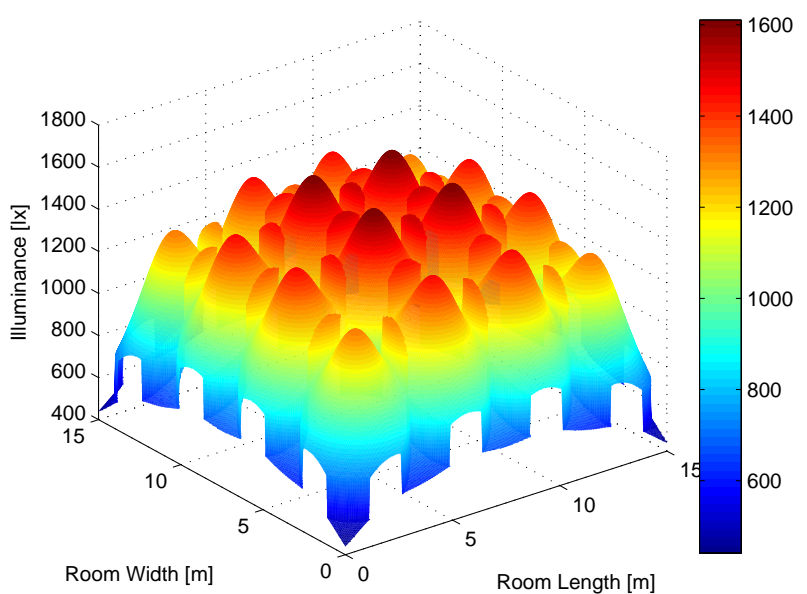

(b)

Fig. 3: (a) Illuminance distribution of a $15 \mathrm{~m} \times 15 \mathrm{~m}$ room covered by $8 \times 8$ uniformly distributed LED-array transmitters at a height of $2.5 \mathrm{~m}$, where the transmitted optical power is 9 W per LED array. Min. 520.2 1x, Max. 1430.0 lx, Ave. 1120.2 1x. (b) Illuminance distribution of the same room covered by $4 \times 4$ uniformly distributed LED-array transmitters at a height of $2.5 \mathrm{~m}$, where the transmitted optical power is $36 \mathrm{~W}$ per LED array and the total power is the same as in (a). Min. 439.0 lx, Max. 1612.9 1x, Ave. 1157.0 1x.

parameters summarized in Table IV into (1)-(3). We draw the illumination surface of the $100 \times 100$ tiles as shown in Figure 3 . Figure 3 shows the illuminance distribution of the room model for different VLC transmitters arrangements. In Figure $3 \mathrm{a}, 8 \times 8$ LED-array transmitters are uniformly distributed on the ceiling, while $4 \times 4$ uniformly distributed transmitters are assumed in Figure 3b. Furthermore, the total transmitted optical power is the same in both settings. The illuminance range is between 300-1500 lx in Figure 3a, which satisfies the lighting standard of a typical room. However, the illuminance may be excessively bright for human eyes in some areas in Figure 3b. since it is as high as 1600 lx. 


\begin{tabular}{|l|r|}
\hline \multicolumn{2}{|c|}{ VLC parameters } \\
\hline Semi-angle at half power $\left(\phi_{1 / 2}\right)$ & $70^{\circ}$ \\
\hline Gain of an optical filter $\left(T_{s}(\psi)\right)$ & 1.0 \\
\hline Refractive index of a lens at a PD $\left(n_{r}\right)$ & 1.5 \\
\hline Modulation bandwidth $(B)[26]$ & $20[\mathrm{MHz}]$ \\
\hline Detection area of a PD $\left(A_{\mathrm{PD}}\right)$ & $1.0\left[\mathrm{~cm}^{2}\right]$ \\
\hline O/E conversion efficiency $(\gamma)$ & $0.53[\mathrm{~A} / \mathrm{W}]$ \\
\hline Half of the receiver's FoV $\left(\psi_{\mathrm{F}}\right)$ & $45^{\circ}$ \\
\hline Optical power to luminous flux conversion factor & $4.2[\mathrm{~mW} / \mathrm{lm}]$ \\
\hline Transmitted optical power per LED array & $9[\mathrm{~W}]$ \\
\hline \hline \multicolumn{2}{|c|}{ Room model parameters } \\
\hline Room size & $15 \mathrm{~m} \times 15 \mathrm{~m} \times 3 \mathrm{~m}$ \\
\hline Height of VLC transmitters & $2.5 \mathrm{~m}$ \\
\hline Distribution of VLC APs & $8 \times 8$ \\
\hline Height of illuminated surface & $0.85[\mathrm{~m}]$ \\
\hline
\end{tabular}

TABLE IV: Simulation parameters.

2) VLC Receiver: PDs are widely used as the receivers in VLC systems. A PD is a semiconductor device, which converts the information-bearing optical radiation into the equivalent electrical signal with the aim of recovering the transmitted information [8]. In particular, the electrical signal power is generated proportionally to the square of the instantaneous received optical power. A relevant parameter to characterize a PD is termed as quantum efficiency, which may be defined as the ratio of the number of the electron-hole pairs ${ }^{2}$ and that of the incident photons in a given time. Apart from the quantum efficiency, the speed of response and the bandwidth of a PD are also essential, which are dependent on the transit time through the depletion regions, the electronic frequency response and the slow diffusion outside the depletion regions, etc. Furthermore, when employing many PDs together as an array, the imaging sensor can also be adopted as the VLC receiver. However, due to its low sampling rate, the achievable rate of an imaging sensor is much lower than that of a standalone PD [14].

\section{B. VLC Channels}

1) General Description of VLC Propagation Model: Figure 4 shows a stylized propagation model of VLC links, including the line-of-sight (LoS) links, the first reflection as well as the high-order reflections. However, the average optical power received from all links, which are reflected more than once, may be negligible according to [31]. In the model of Figure 4, a VLC transmitter TX located on the ceiling transmits data to a VLC receiver RX. The angles of irradiance in the LoS link and the first-reflection link are denoted as $\phi_{d}$ and $\phi_{r}$, while correspondingly the angles of incidence in each link are denoted as $\psi_{d}$ and $\psi_{r}$, respectively. The LoS optical channel's total DC attenuation $h_{d}[u, a]$ from a VLC transmitter TX to the receiver RX is given by [35], [36]

$$
h_{d}[u, a]=\left\{\begin{array}{lr}
\frac{(w+1) \cdot A_{\mathrm{PD}}}{2 \pi d_{a, u}^{2}} \cdot \cos ^{w}\left(\phi_{d}\right) \cdot T_{s}\left(\psi_{d}\right) \cdot g\left(\psi_{d}\right) \cdot \cos \left(\psi_{d}\right) \\
\psi_{d} \leq \psi_{\mathrm{F}}, \\
\psi_{d}>\psi_{\mathrm{F}},
\end{array}\right.
$$

\footnotetext{
${ }^{2}$ An electro-hole is the phenomenon that an electron leaves its position in an atom or atomic lattice.
}

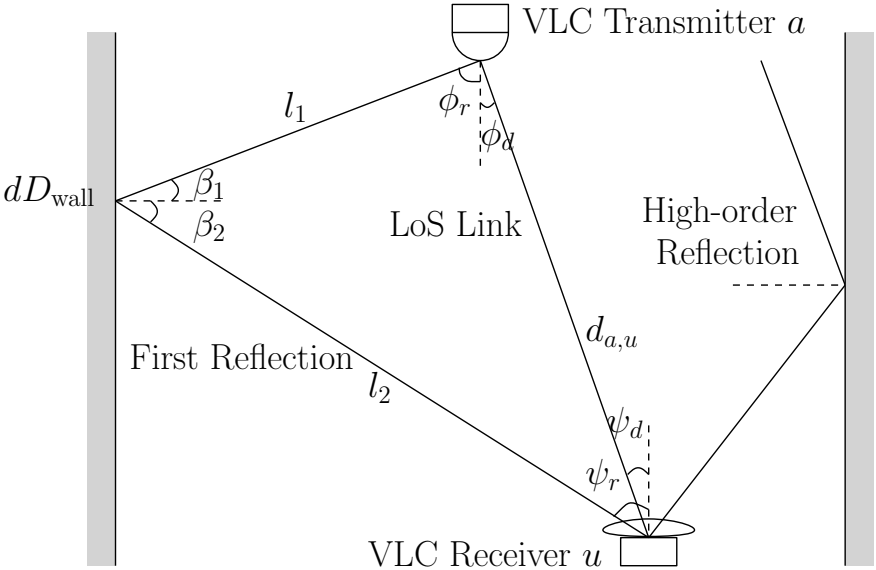

Fig. 4: Propagation model of VLC links, including the LoS links, the first reflection as well as the $k$ th reflection. However, the average received optical power from all links, which are reflected more than once, may be negligible according to [31].

where the Lambert index $w$ depends on the semi-angle $\phi_{1 / 2}$ at half-illuminance of the source, which is given by $w=$ $-1 / \log _{2}\left(\cos \phi_{1 / 2}\right) . A_{\mathrm{PD}}$ is the detector's physical area for a PD and $\psi_{\mathrm{F}}$ represents half of the receiver's field-of-view (FoV). In a direct LoS path, the irradiant angle equals to the incident angle, where both the transmitter and the receiver point vertically. As shown by Figure 4 , we have $\phi_{d}=\psi_{d}$. Still referring to (4), $T_{s}\left(\psi_{d}\right)$ and $g\left(\psi_{d}\right)$ denote the gain of the optical filter and of the optical concentrator employed, respectively, while $g\left(\psi_{d}\right)$ can be written as [35]

$$
g\left(\psi_{d}\right)= \begin{cases}\frac{n_{r}^{2}}{\sin ^{2} \psi_{d}}, & 0 \leq \psi_{d} \leq \psi_{\mathrm{F}}, \\ 0, & \psi_{d}>\psi_{\mathrm{F}},\end{cases}
$$

where $n_{r}$ is the refractive index of a lens at a PD.

Furthermore, according to [31], when the incidence angle $\psi_{r}$ is no larger than the FoV, the channel's DC attenuation $d h_{r}[u, a]$ on the first reflection is given by

$$
\begin{aligned}
d h_{r}[u, a]= & \frac{(w+1) \cdot A_{\mathrm{PD}}}{2 \pi^{2} l_{1}^{2} l_{2}^{2}} \cdot \rho \cdot d D_{\text {wall }} \cdot \cos ^{w}\left(\phi_{r}\right) \cdot \cos \left(\beta_{1}\right) . \\
& \cos \left(\beta_{2}\right) \cdot T_{s}\left(\psi_{r}\right) \cdot g\left(\psi_{r}\right) \cdot \cos \left(\psi_{r}\right),
\end{aligned}
$$

where $l_{1}$ represents the distance between the transmitter TX and a reflective point, while $l_{2}$ is the distance between this point and the receiver RX. The reflectance factor and the reflective area are denoted by $\rho$ and $d D_{\text {wall }}$, respectively. Additionally, $\beta_{1}$ and $\beta_{2}$ represent the irradiance angles to the reflective point and to the receiver, respectively. Our parameter values are summarized in Table IV.

2) VLC Channel Models: The basic channel model of a )VLC system is given by

$$
y(t)=x(t) \otimes h(t)+n(t),
$$

where $x(t)$ and $y(t)$ denote the transmitted and received signal, respectively. $h(t)$ is the channel's impulse response (CIR) and $n(t)$ represents the total noise. $\otimes$ is the convolution operator. The CIR $h(t)$ may be used to describe the channel 
characteristics of VLC. Most of the existing VLC channel models are mainly based on the infrared channel models. As reported in [26], [37]-[40], valuable research has been dedicated to modelling the VLC channel by considering some of the practical VLC channel characteristics. In particular, multipath propagation imposed by reflections results in spreading of the received signal, and the power delay profile (PDP) can be used to analyze the effect of multipath dispersion between transceivers. One of the widely used VLC channels was proposed in [37], [38] by Lee et al., where the multipath $\mathrm{PDP}$ is given as

$$
h(t)=\sum_{n=1}^{N_{\mathrm{LED}}} \sum_{k=0}^{\infty} h^{(k)}\left(t ; p_{n}\right),
$$

with $N_{\text {LED }}$ denoting the total number of LEDs and each LED is assumed to transmit the same power. Furthermore, $p_{n}$ is the power spectral distribution of the $n$th LED. The response after the $k$ th-reflection to the $n$ th-LED source is [37]

$$
\begin{aligned}
h^{(k)}\left(t ; p_{n}\right)=\int_{S} & {\left[L_{1} \cdot L_{2} \cdot \ldots \cdot L_{k+1} \cdot \Gamma_{n}^{(k)} \cdot \operatorname{rect}\left(\frac{\theta_{k+1}}{\psi_{\mathrm{F}}}\right) .\right.} \\
& \left.\delta\left(t-\frac{d_{1}+d_{2}+\ldots+d_{k+1}}{c}\right)\right] d_{A_{\text {ref }}}, k \geq 1,
\end{aligned}
$$

where $L_{k}$ denotes the path-loss of the $k$ th path and we have

$$
\begin{aligned}
L_{1} & =\frac{A_{\mathrm{ref}} \cdot(w+1) \cdot \cos ^{w}\left(\phi_{1}\right) \cdot \cos \left(\psi_{1}\right)}{2 \pi d_{1}^{2}}, \\
L_{2} & =\frac{A_{\mathrm{ref}} \cdot \cos \left(\phi_{2}\right) \cdot \cos \left(\psi_{2}\right)}{\pi d_{2}^{2}}, \ldots, \\
L_{k+1} & =\frac{A_{\mathrm{PD}} \cdot \cos \left(\phi_{k}\right) \cdot \cos \left(\psi_{k+1}\right)}{\pi d_{k+1}^{2}} .
\end{aligned}
$$

In (10), $A_{\text {ref }}$ is the reflecting area and the integration in (9) is performed with respect to the surface $S$ of all reflectors, while $d_{k}$ denotes the length of the $k$ th path. The rectangular function $\operatorname{rect}(x)=1$, if $|x| \leq 1$; otherwise, $\operatorname{rect}(x)=0$. Furthermore, the reflected power $\Gamma_{n}^{(k)}$ is defined as

$$
\Gamma_{n}^{(k)}=\int_{\lambda} p_{n}(\lambda) \cdot \rho_{1}(\lambda) \cdot \rho_{2}(\lambda) \cdot \ldots \cdot \rho_{k}(\lambda) d \lambda,
$$

where $\rho_{k}(\lambda)$ is the spectral reflectance. The PDP of LoS propagation is given by [37]

$$
h^{(0)}\left(t ; p_{n}\right)=L_{0} \cdot P_{n} \cdot \operatorname{rect}\left(\frac{\theta_{0}}{\psi_{\mathrm{F}}}\right) \cdot \delta\left(t-\frac{d_{0}}{c}\right),
$$

where

$$
\begin{aligned}
L_{0} & =\frac{A_{\mathrm{PD}} \cdot(w+1) \cos ^{w}\left(\phi_{0}\right) \cdot \cos \left(\psi_{0}\right)}{2 \pi d_{0}^{2}}, \\
P_{n} & =\int_{\lambda} p_{n}(\lambda) d \lambda .
\end{aligned}
$$

Lee et al. [37] considered the power spectral distribution of the reflected LED light and the wavelength-dependent reflectance of VLC channels as characterized by (9)-(12).

Furthermore, there are also some other VLC channel models in the literature. The classic ceiling-bounce model developed by Carruthers and Kahn [41] for indoor infrared systems was introduced into VLC systems by Rajbhandari et al. [39]. Owing to its simplicity and excellent matching with the measured data, the ceiling-bounce model is the most popular one in simulations [8]. However, this channel model only characterizes two parameters, i.e. the optical path loss and the root mean square delay spread, which may not be suitable for discussing the impact of other channel parameters. Relying on the Monte-Carlo ray tracing simulation and the assumption of the Lambert-Phong reflection pattern, Ding and Ke [40] proposed a new VLC multipath channel model, where the LoS and the scattered path are considered separately. Another channel model widely used in infrared systems is the integrating-sphere model of [42], which has also been employed in VLC systems [43], where the diffuse signal gain is assumed to be constant everywhere in the room. The different VLC channel models are compared in Table V.

3) Shadowing Effect: Since visible light cannot propagate through most of the objects in indoor environments, it is essential to study its shadowing effect. Komine and Nakagawa [44] characterized the shadowing effects in indoor VLC systems caused by pedestrians with the aid of simulations. They found that the shadowing effects may be reduced, hence reducing the outage probability and the blocking probability, by optimizing the number of LEDs. Apart from this preliminary work, Farahneh et al. [45] modelled the shadowing effects encountered in visible light scenarios by a bimodal Gaussian distribution and derived the probability of error for low, moderate and severe shadowing conditions. However, there is still a paucity of studies on the general shadowing model of indoor VLC systems, although recently some valuable studies characterized the impact of the recievers' mobility and of human behaviour on the VLC system's performance [46], [47]. Chvojka et al. [46] investigated the changes in channel characteristics caused by blocking and showed that the corridor model is the most robust, while the office/hall model is the most vulnurable one against people's movements. Furthermore, Bas et al. [47] highlighted the impact of the moving human body on a VLC link, which is of more significance in VLC systems.

\section{PHY Techniques}

1) Modulation Schemes: Modulation schemes constitute one of the most important physical-layer techniques in communication systems. This is especially true when jointly considering indoor illumination requirements, since the modulated signals can be used to switch on/off the LEDs, which conveys the on-off pattern-based binary information to the receiver. Since LEDs emit incoherent light, where photons have different wavelength and phase, it is an open challenge to collect appreciable signal power in a single electromagnetic mode in a practical low-cost VLC system. Hence, often the intensity modulation direct detection (IM-DD) scheme is employed, where the transmitted signal modulates the instantaneous optical power of the LEDs. Hence, IM-DD constitutes an attractive scheme and has been widely used in VLC systems.

Let us now elaborate a little further. The simple on-off keying $(\mathrm{OOK})$ technique is realized by switching a LED, where the binary signals are transmitted as the presence or absence of 


\begin{tabular}{|l|l|l|l|l|}
\hline \multicolumn{1}{|c|}{ Model } & \multicolumn{1}{|c|}{ Basis } & Propagation paths & \multicolumn{1}{c|}{ Advantages } & Disadvantages \\
\hline General model [31] & $\begin{array}{l}\text { Infrared channel } \\
\text { model, simplified } \\
\text { assumption }\end{array}$ & $\begin{array}{l}\text { LoS and 1st re- } \\
\text { flected path }\end{array}$ & Simple calculation & Higher-order reflections ignored \\
\hline Lee et al. [37], [38] & $\begin{array}{l}\text { Infrared channel } \\
\text { model }\end{array}$ & $\begin{array}{l}\text { LoS and } \\
\text { reflected paths }\end{array}$ & $\begin{array}{l}\text { Considering power spectral distribution of LED } \\
\text { light and the wavelength-dependent reflectance }\end{array}$ & No discussion on the number of LEDs \\
\hline Rajbhandari et al. [39] & $\begin{array}{l}\text { Ceiling bounce } \\
\text { model }\end{array}$ & Scattered links & Time-invariant channel, simple expression & Considering few parameters \\
\hline Ding and Ke [40] & $\begin{array}{l}\text { Ray tracing simula- } \\
\text { tion, Lambert-Phong } \\
\text { reflection pattern }\end{array}$ & $\begin{array}{l}\text { Multipath chan- } \\
\text { nels }\end{array}$ & $\begin{array}{l}\text { Calculation linear to the number of reflections, } \\
\text { suitable in complex environments with blockage }\end{array}$ & Some parameters not defined clearly \\
Zeng et al. [43] & $\begin{array}{l}\text { Integrating-sphere } \\
\text { model }\end{array}$ & $\begin{array}{l}\text { LoS and dif- } \\
\text { fused paths }\end{array}$ & Constant diffuse signal gain & Lack of justification for VLC systems \\
\hline
\end{tabular}

TABLE V: Comparison of different VLC channel models, reproduced from [16].

light [31], [48]. In [49], an aggregate parallel data transmission rate of $1.5 \mathrm{~Gb} / \mathrm{s}$ was achieved by employing OOK modulation. Furthermore, various pulse position modulation (PPM) schemes have been introduced into VLC systems for achieving different design objectives [50]-[52], where the basic concept is to map the modulated signal into $2^{M}$ legitimate time-slots for $M$-ary PPM [53]. Another bandwidth-efficient baseband modulation technique is constituted by pulse amplitude modulation (PAM) [35], which was also invoked and evaluated in VLC systems [26], [54]. However, the signals modulated by multiple intensity levels may suffer from nonlinear distortions due to the LEDs intensity-dependent luminous efficacy [13]. On the other hand, in order to provide high data rates, multiplecarrier techniques have also been applied in VLC systems, such as the quadrature amplitude modulation (QAM)-based discrete multitone (DMT) transmission technique [26].

Apart from the widely known modulation schemes, some unique modulation techniques have also been developed for VLC systems, including colour shift keying (CSK) [55][58], generalized colour modulation (GCM) [59], [60], optical orthogonal frequency division multiplexing (OOFDM) [61][65], etc. Explicitly, the CSK scheme using multi-chip LEDs was standardized in the IEEE 802.15.7 recommendation [66], which may be a potential modulation scheme for future VLC systems, although it relies on sophisticated implementation. In [57], Jiang et al. investigated an uncoded $M$-CSK scheme relying on a joint maximum likelihood hard-detection based VLC system, where both simulation-based and analytical biterror-rate (BER) results were derived. By contrast, a colourindependent GCM scheme was proposed in [59], which had the advantages of flicker-free operation, accurate dimming control and the ability to function independently of the number of LEDs at the transmitter or the PDs at the receiver [60]. Moreover, there are three popular types of OOFDM proposed for VLC systems, i.e. asymmetrically clipped OOFDM (ACO-OFDM) [61], [67], DC biased OOFDM (DCO-OFDM) [62], [68] as well as unipolar OFDM (U-OFDM) [63]. The milestones of popular modulation schemes employed in VLC systems are summarized in the timeline of Table VII. Furthermore, the advantages and disadvantages of the major modulation schemes are listed in Table VI.

2) Dimming Control and Flicker Mitigation: According to [32], different levels of illuminance are required in different scenarios. Owing to the fact that an LED can be dimmed to an arbitrary level, integrating dimming control into VLC systems

\begin{tabular}{|c|l|l|}
\hline Scheme & \multicolumn{1}{|c|}{ Advantages } & \multicolumn{1}{c|}{ Disadvantages } \\
\hline OOK & Simple implementation & $\begin{array}{l}\text { Low data rate, slow flicker- } \\
\text { ing }\end{array}$ \\
\hline PPM & Simple implementation & Low spectral efficiency \\
\hline CSK & High data rate & $\begin{array}{l}\text { Sophisticated implementa- } \\
\text { tion }\end{array}$ \\
\hline OOFDM & High data rate & $\begin{array}{l}\text { Clipping distortion, limited } \\
\text { support for dimming }\end{array}$ \\
\hline
\end{tabular}

TABLE VI: Advantages and disadvantages of popular VLC modulation shcemes.

is required for the sake of saving energy [48]. The light should be dimmable arbitrarily in VLC systems, without the communications becoming interrupted, which means that the data should be modulated in such a way that any desired level of dimming is supported [14]. Numerous valuable modulation schemes supporting dimming control have been proposed for the LEDs, such as the variable OOK [48], [72], variable PPM [73], pulse width modulation with DMT [74], etc.

When the light intensity changes, it should be flickering above the human eyes' fusion frequency, so that the human eye cannot perceive it. Otherwise, serious physiological problems may be caused. Hence, the speed of the changes in light intensity, termed as flickering, should be carefully considered when designing modulation schemes for VLC systems. As suggested by the IEEE standard [66], flickering faster than $200 \mathrm{~Hz}$ can avoid harmful effects to the human eye. In order to mitigate slow flickering, run length limited codes [48] have been incorporated into the VLC modulation schemes for avoiding long runs of $0 \mathrm{~s}$ and $1 \mathrm{~s}$, which is believed to be the most common reason of slow flickering.

\section{VLC NETWORKS}

Beyond point-to-point links, VLC is also considered as a new member in the small-cell family of the HetNets landscape for complementing the overloaded RF band [22]. In this section, the layout of the visible-light-aided networks is investigated, ranging from a regular network-centric cell-layout associated with different frequency reuse (FR) patterns to the radically new user-centric (UC) cluster formation employing advanced transmission schemes. Furthermore, the family of multiuser techniques such as multiuser signal processing, scheduling and resource management are reviewed. 


\begin{tabular}{|c|c|}
\hline Year & Modulation schemes \\
\hline 1993 & $\begin{array}{l}\text { Huang et al. [69] employed subcarrier PSK modulation in an atmospheric optical communica- } \\
\text { tion system. }\end{array}$ \\
\hline 2004 & $\begin{array}{l}\text { Komine et al. [31] analysed a typical VLC system relying on white LEDs and they considered } \\
\text { the classic OOK modulation scheme. }\end{array}$ \\
\hline 2006 & Armstrong and Lowery [70] proposed ACO-OFDM and analysed its performance. \\
\hline 2007 & Li et al. [61] investigated the channel capacity of ACO-OFDM. \\
\hline 2008 & $\begin{array}{l}\text { Grubor } \text { et al. [26] investigated the performance of the room model of [31] relying on QAM- } \\
\text { based DMT-aided transmission. }\end{array}$ \\
\hline 2011 & The CSK scheme using multi-chip LEDs was standardized in IEEE 802.15.7 [66]. \\
\hline 2012 & Tsonev et al. [63] proposed U-OFDM for optical wireless systems. \\
\hline \multirow{2}{*}{2013} & Das et al. [59] proposed a colour-independent GCM scheme. \\
\hline & $\begin{array}{l}\text { Dissanayake and Armstrong [71] compared the performance of ACO-OFDM, DCO-OFDM and } \\
\text { asymmetrically clipped DC biased OOFDM. }\end{array}$ \\
\hline 2014 & $\begin{array}{l}\text { Noshad and Brandt-Pearce [51], [52] proposed the expurgated PPM scheme for providing a } \\
\text { wide range of peak to average power ratios needed for dimming. }\end{array}$ \\
\hline 2015 & $\begin{array}{l}\text { Jiang et al. [57] investigated an uncoded } M-\text { CSK scheme and provided both the simulation- } \\
\text { based and the analytical BER results. }\end{array}$ \\
\hline
\end{tabular}

TABLE VII: A brief summary of popular modulation schemes reported in VLC systems.

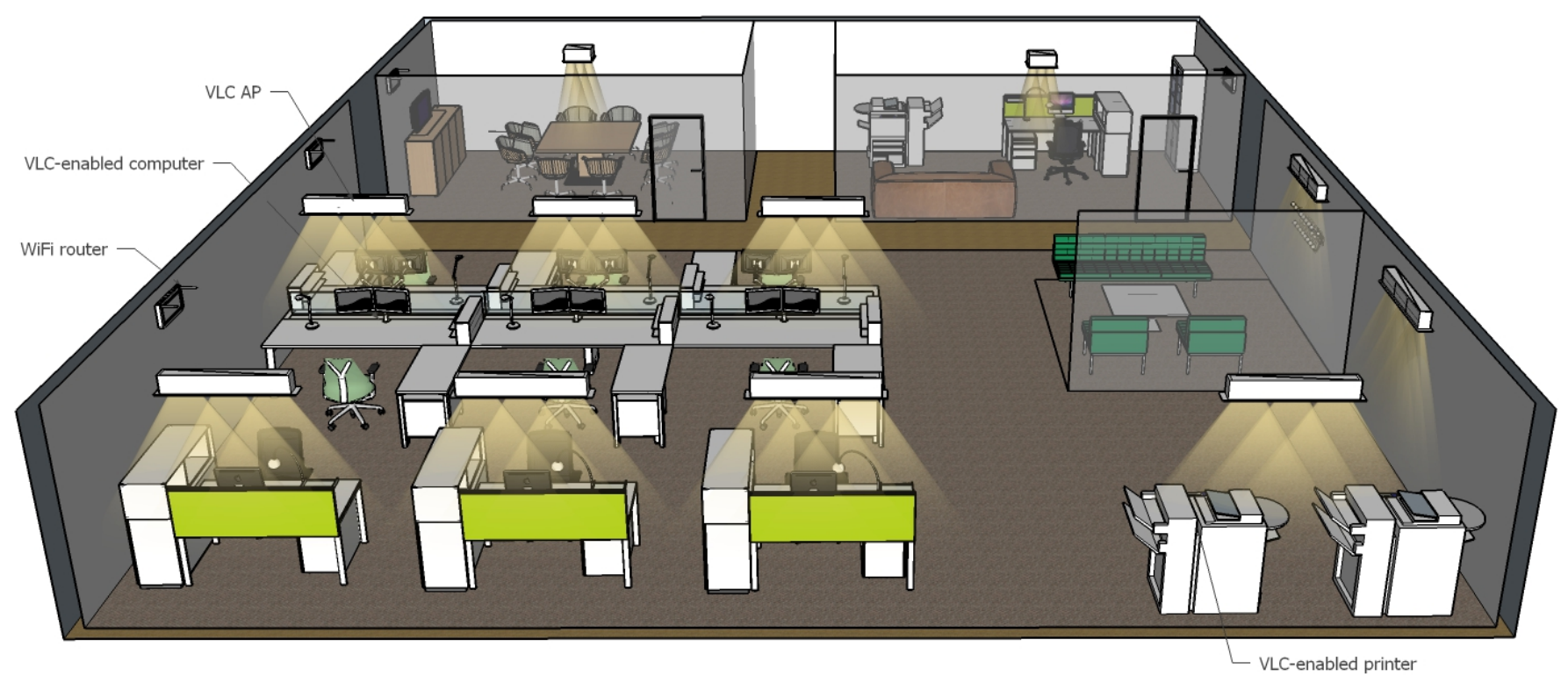

Fig. 5: A stylized indoor VLC system.

\section{A. Network-Centric Optical Attocells}

In a typical VLC down-link (DL) network, each optical access point (AP) illuminates only a small confined cell, as shown in Figure 5. The coverage of the single-AP optical cell is usually limited to room-size $(\sim \mathrm{m})$ and it is considerably smaller than the coverage of the traditional RF cells $(\sim \mathrm{km})$, which is referred to as an optical attocell [75], where atto means $10^{-18}$ in physics and mathematics. The concept of optical attocells is borrowed from the RF attocell, which was originally announced by the company Ubiquisys to represent the personal femtocell in 2011. Considering a specific optical attocell, its coverage is dependent both on the illumination requirements as well as on the FoV of the VLC receivers. Relying on these optical attocells, the indoor VLC network may be operated by assuming the full functionality of a RF cellular DL system. Note that the optical attocell is merely limited to the single-AP optical cell and we focus on the downlink transmission in the discussion of this section.
When there are multiple optical attocells in a VLC network, the inter-cell interference (ICI) imposed both by the LoS ray as well as by the non-LoS (NLoS) links has to be carefully treated, since the performance may become dramatically degraded at the cell edge due to ICI [76]. This degradation itself both in terms of the signal-to-interference-plus-noise ratio (SINR), the BER, the bandwidth efficiency, etc. and consequently vertical handovers may be frequently activated in VLC-based HetNets [77]. In order to reduce the effect of the ICI and to improve the performance, valuable research has been dedicated to

- the initial design of the optical APs deployment [78][80],

- the frequency planning within the optical spectrum available [81]-[84], as well as

- the cell coordination between the multiple neighbouring optical attocells [85], [86]. 


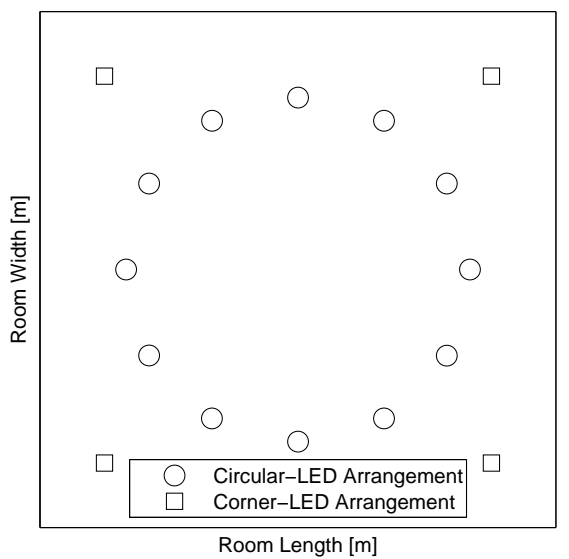

Fig. 6: A novel VLC AP arrangement was advocated in [78], where 12 LED lamps constitute a circle and 4 LED lamps are placed in the corners, which are referred to here as the circularLED arrangement and corner-LED arrangement, respectively.

1) Novel Design of VLC AP Arrangements: To elaborate a little further, the ICI avoidance methods were considered at the preliminary design stage of the entire VLC-network design cycle by the authors of [78]-[80], [83]. A novel VLC AP arrangement was advocated in [78], where 12 LEDs constitute a circle and 4 LEDs are placed in the corners, which are referred to here as the circular-LED arrangement and cornerLED arrangement, respectively, as shown in Figure 6. Compared to the arrangement of [31], where a single LED lamp is placed in the centre of the ceiling illuminating the entire room with the same total emitted optical power as in Figure 6, the arrangement proposed in [78] provides a much lower variance of the received signal-to-noise ratio (SNR) and of the received power at different locations in the room. Furthermore, the radius of the circle constituted by the circular-LED arrangement may be optimized according to the receivers' specific locations as well as to the various layouts of the room considered. The optimization problem of the LED-lamp placement was also formulated and solved in [79] for the sake of maximising the average area spectral efficiency [bits $\left./ \mathrm{s} / \mathrm{Hz} / \mathrm{m}^{2}\right]$ [87]. Upon studying the optimum layout of two LED lamps with different FoV of the receiver, they found that the average area spectral efficiency is degraded, when imposing the classic lighting requirements on the communication performance maximization problem. Moreover, instead of optimizing the placement of the LED lamps, Guan et al. [80] investigated two LED deployment schemes, i.e. the single-LED-based deployment scheme and the array-LED-based deployment scheme. They used the classic particle swarm optimization algorithm, in order to minimize the average outage area. Both of their proposed schemes are superior to the conventional random deployment scheme.

2) Non-unity Frequency Reuse: Similar to the traditional cellular design principles, FR constitutes an appealingly simple solution for ICI mitigation. Figure $7 \mathrm{a}$ and Figure $7 \mathrm{~b}$ show the neighboring optical attocells employing a unity frequency reuse (UFR) and a FR factor of two (FR-2), respectively. Similarly to classic RF cells, it is plausible that the ICI-infested areas are reduced, when a non-unity FR factor is used. Since the first-tier neighboring cells contribute most of the ICI, while the second-tier cells generally have a negligible influence, using FR-2 strikes an attractive tradeoff in terms of providing an adequate SINR at the cell edge, unless the receiver's FoV is extremely wide or there is a large cell overlap. Marsh and Kahn [81] contributed one of the early studies investigating FR schemes conceived for optical communications, where an indoor infrared wireless communication system employing fixed-channel reuse for optical base stations was examined. In contrast to the static FR planning scheme, the resources are dynamically reused relying on a self-organizing allocation mechanism in [82] and a scalable orthogonal frequencydivision multiplexing access (OFDMA)-based VLC system is proposed in [83]. More recently, the Chen et al. [84] designed a pair of fractional FR schemes for the DCO-OFDM-based optical attocell based networks, where strict fractional FR and soft FR were employed. However, although FR planning is an effective solution, the system has to obey the classic trade-off between having reduced bandwidth efficiency and an improved SINR. Furthermore, an inherent disadvantage of FR is that switching between optical frequencies every few meters degrades the user experience [22].

3) Advanced Transmission Scheme: On the other hand, advanced cooperative transmission by multiple LEDs may be conceived by relying on the elegant time-of-arrival synchronization advocated in [85], where multiple LEDs transmit simultaneously to the same receiver. In [86], three modulation techniques, namely OOK, PPM and pulse width modulation, were employed in the neighbouring optical cells and this scheme was expected to achieve a data rate of $71 \mathrm{Mb} / \mathrm{s}$, whilst providing seamless coverage. More recently, the philosophy of combined transmission (CT) techniques was employed in multiuser VLC networks in [76] and a similar concept termed as a joint transmission scheme was introduced in [88]. In this arrangement, each individual VLC AP of a multi-AP cell conveys the same information on the same visible carrier frequency in their overlapping areas. Figure $7 \mathrm{c}$ shows a pair of merged 2-AP cells (AB and CD) employing CT (CT-2). Thus the ICI encountered in the overlapping coverage areas of cell A and cell B (or cell C and cell D) turns into desired signal and the SINR is significantly improved at the cell edge compared to the regular UFR transmission scheme. Although the SINR is indeed enhanced, CT results in a reduced bandwidth efficiency, since only a single user is served at any instant by several APs in the overlapping area within a merged cell. In order to eliminate the bandwidth efficiency reduction imposed by $\mathrm{CT}$, multiuser vectored transmission (VT) techniques were introduced [22], [76] in the context of merged multi-AP cells, where multiple UEs are supported at the same time. As an example, observe in Figure 7d that the merged 2-AP cell (VT2) can simultaneously serve two UEs like a $(2 \times 2)$-element multi-input multi-output (MIMO) system by employing VT techniques.

4) Bandwidth Efficiency: To gain further quantitative insights, Figure 8 illustrates the bandwidth efficiency surface of 


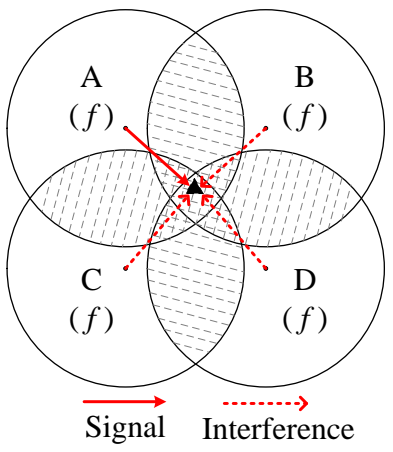

(a) UFR

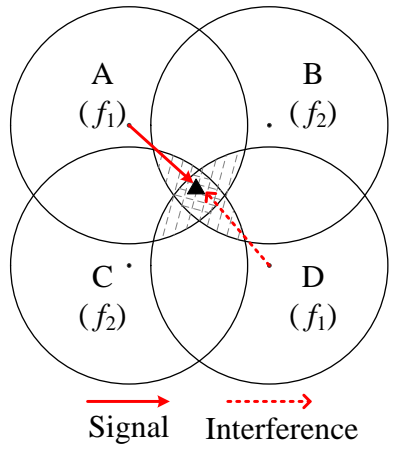

(b) FR-2

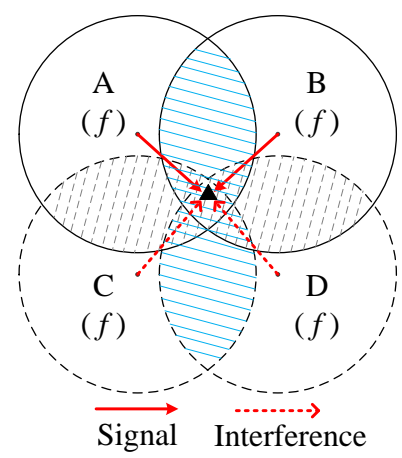

(c) CT-2

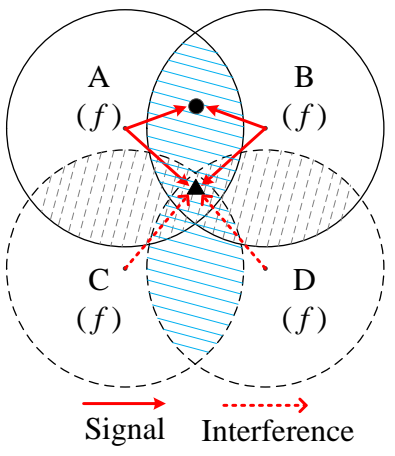

(d) VT-2

Fig. 7: Different network-centric cell formations [76]. (a) is a regular cell formation, (b) has a FR factor of two, (c) represents two merged 2-AP cells with CT and (d) shows two merged 2-AP cells using VT. The triangle and circle denote certain points of reception. The shaded areas covered with dotted lines represent the ICI imposed by the LOS ray of neighbouring cells at the cell edge. The shaded areas covered by solid lines represent the overlapping areas within the merged 2-AP cells. In (a), (c) and (d), the entire frequency band $f$ is used by each small cell, while in (b) orthogonal frequencies $f_{1}$ and $f_{2}$ are employed by neighbouring cells, where we have $f_{1}=f_{2}=f / 2$.

both optical single-AP attocells using different FR patterns as well as that of merged multi-AP VLC cell formations using advanced transmission techniques. The classic bandwidth efficiency $\eta$ is calculated as

$$
\begin{array}{ll}
\eta_{\mathrm{UFR}}=\log _{2}\left(1+\xi_{\mathrm{UFR}}\right) ; & \eta_{\mathrm{FR}-2}=\frac{1}{2} \log _{2}\left(1+\xi_{\mathrm{FR}-2}\right) ; \\
\eta_{\mathrm{CT}}=\hbar \log _{2}\left(1+\xi_{\mathrm{CT}-2}\right) ; & \eta_{\mathrm{VT}}=\log _{2}\left(1+\xi_{\mathrm{VT}}\right) ;
\end{array}
$$

where $\xi$ denotes the received SINR, while $\hbar$ accounts for the resource-efficiency reduction of $\mathrm{CT}^{3}$. The achievable data rate is given by $B \cdot \eta$, where $B$ denotes the bandwidth. Our simulation parameters characterizing the VLC APs are summarized in TABLE IV. Compared to UFR characterized in Figure 8a, FR-2 substantially reduces the ICI-contaminated areas, hence significantly increasing the area spectral efficiency (ASE), as shown in Figure 9a. The merged 2-AP cells seen in Figure 8c and Figure 8d also improve the ASE compared to UFR, as suggested in Figure 9a. However, neither CT2 nor VT-2 constitutes an attractive solution for our simulation configuration, since FR-2 is capable of supporting an increased bandwidth efficiency at a reduced implementational complexity ${ }^{4}$. By contrast, when combining more VLC APs into a single cell, a substantial ASE improvements can be achieved with the aid of VT, as shown both in Figure 8e-8f as well as in Figure 9a. Furthermore, Figure 9b-9d exhibit the communication coverage holes of the UFR, FR-2 and VT-16 schemes, respectively. During mobility, the smaller the area of

${ }^{3}$ In Figure $8 \mathrm{c}, \hbar$ is given by

$$
\hbar=\frac{1}{2} \cdot \frac{D_{s}}{D_{m c}}+\frac{D_{m c}-D_{s}}{D_{m c}},
$$

where $D_{s}$ and $D_{m c}$ denote the area of the shaded areas within the merged 2 -AP cell and the total area of the merged cell, respectively.

${ }^{4}$ In [76], the FR-2 scheme provides the lowest bandwidth efficiency, since the simulation parameters are different, although the analysis method is the same. Hence, the cell formation should be designed according to the specific room configurations. However, the performance analysis method is a generic one. coverage holes, the lower the dropping probability becomes. Compared to the UFR scheme characterized in Figure 9b, both the FR-2 and the VT-16 schemes are capable of reducing the area of coverage holes, but VT-16 supports a higher bandwidth efficiency than FR-2. It is plausible that if a total of 64 VLC APs are merged into a single 64-AP cell employing the VT64 scheme, there will be no coverage holes in the middle of the room. However, this is achieved at an increased signal processing cost.

The milestones of the ICI mitigation schemes in VLC systems are summarized in the timeline of Table VIII. The advantages and disadvantages of different ICI mitigation schemes are listed in Table IX. Broadly speaking, most techniques conceived for ICI avoidance and performance improvement in VLC networks were designed from a network-centric perspective, where fixed-shape regular optical attocells were investigated. By contrast, the amorphous user-specific cluster formation philosophy exemplified in Figure 12 is designed from the user-centric perspective discussed in the next subsection.

Remarks: In order to provide a comprehensive practical guidance considering the performance of the general VLC network, the statistics of the achievable SINR may be derived. Similar to the conventional RF cellular networks, the SINR statistics are deemed to be an important metric for characterising the service quality [92], in terms of coverage/outage probability, data rate, etc. For example, the coverage probability/cell boundary of the optical cell may be defined as $\mathbb{P}[\operatorname{SINR}>T]$, which is the probability that a randomly chosen user can achieve a target SINR $T$ or the average fraction of the network area that has adequate "coverage" at any time [93]. The achievable SINR is influenced by various issues, for instance, the available output signal power, the channel characteristics, etc. One of the most important factors is the deployment model of the optical APs. Using an appropriate model is essential for deriving analytically tractable expressions for the SINR 


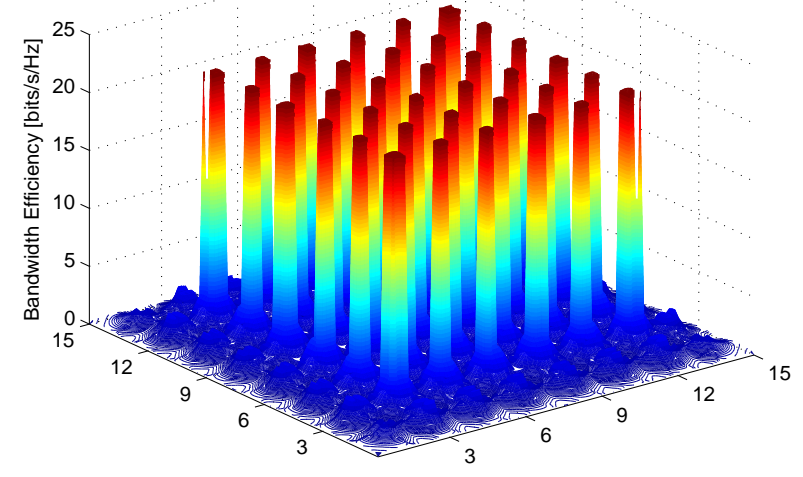

(a) UFR

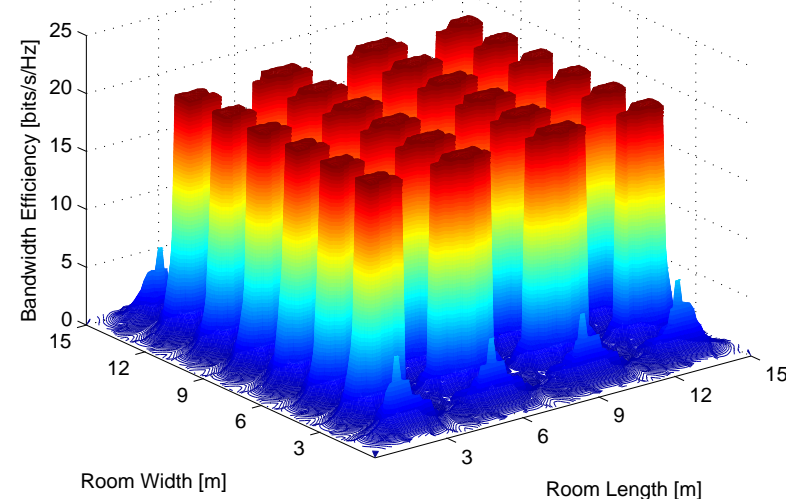

(c) CT-2

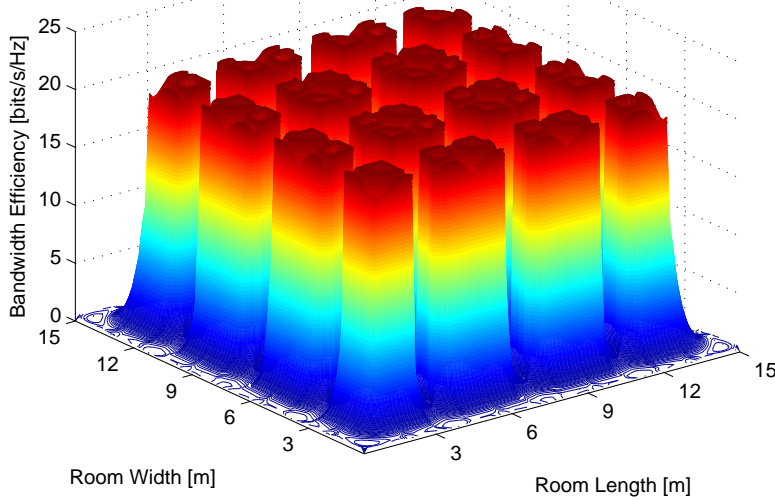

(e) VT-4

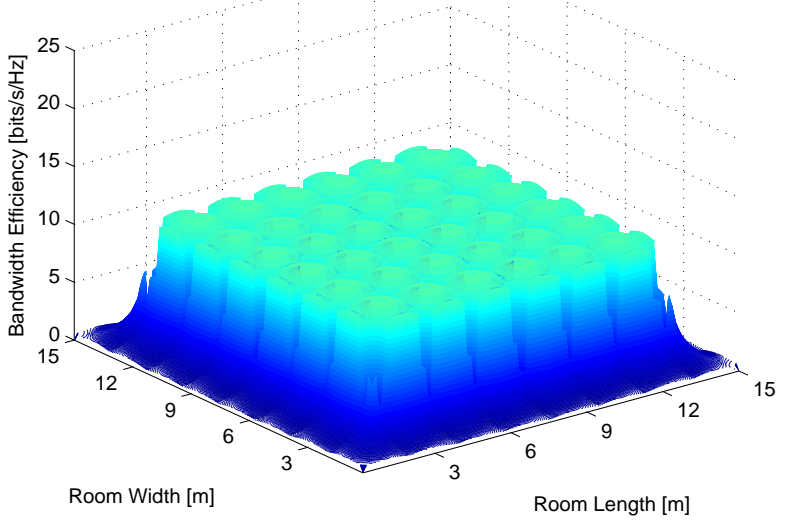

(b) FR-2

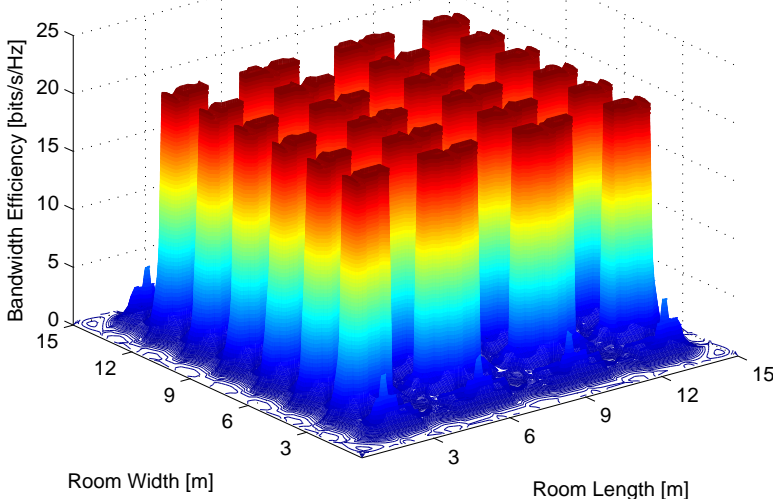

(d) VT-2

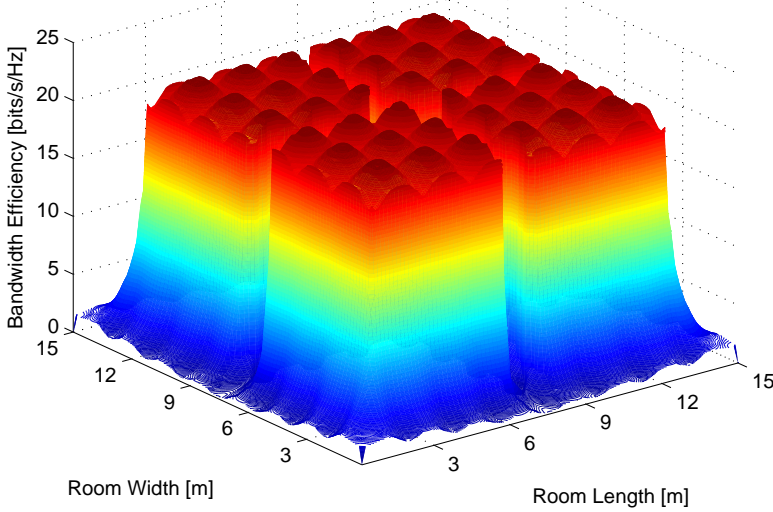

(f) VT-16

Fig. 8: Bandwidth efficiency surface of different VLC cell formations. (a) is a regular UFR cell formaiton. (b) has a FR factor of two. (c) represents a merged 2-AP cells with CT. (d)-(f) shows the merged 2-AP, 4-AP and 16-AP cells with VT, respectively. 


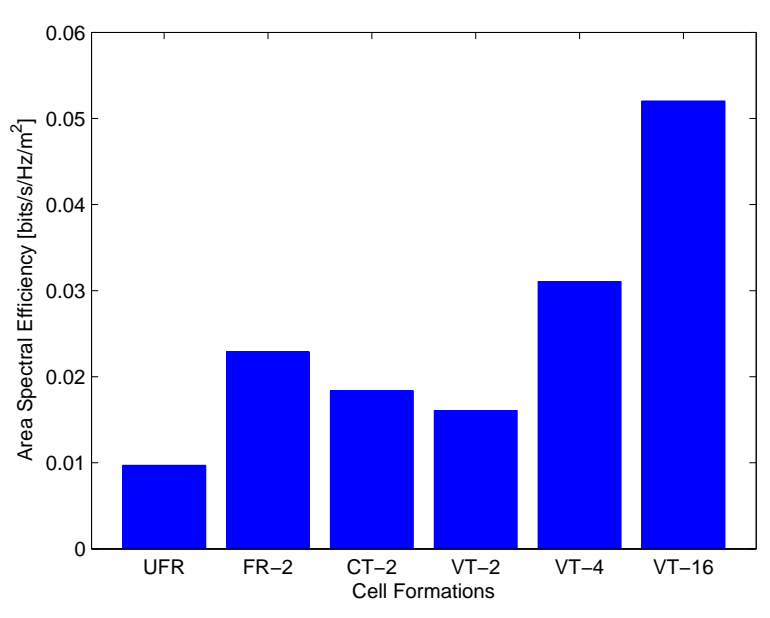

(a) ASE

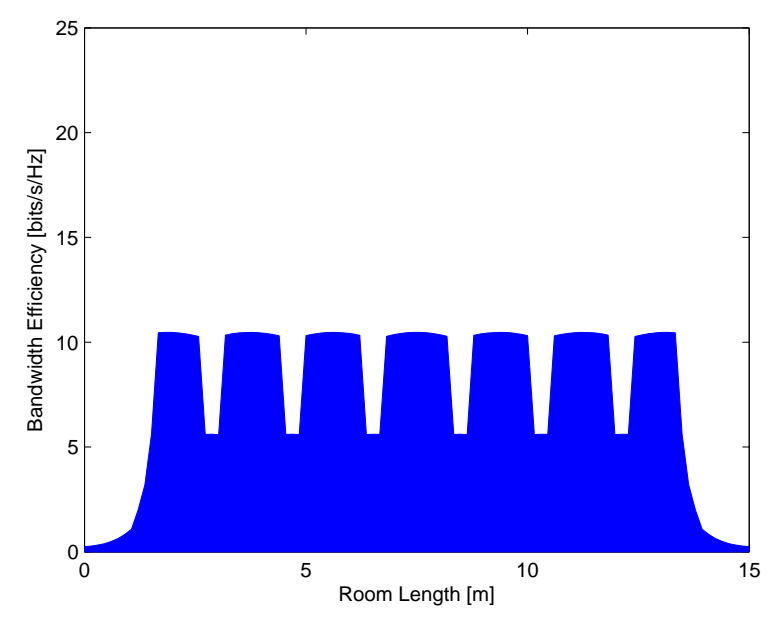

(c) FR-2

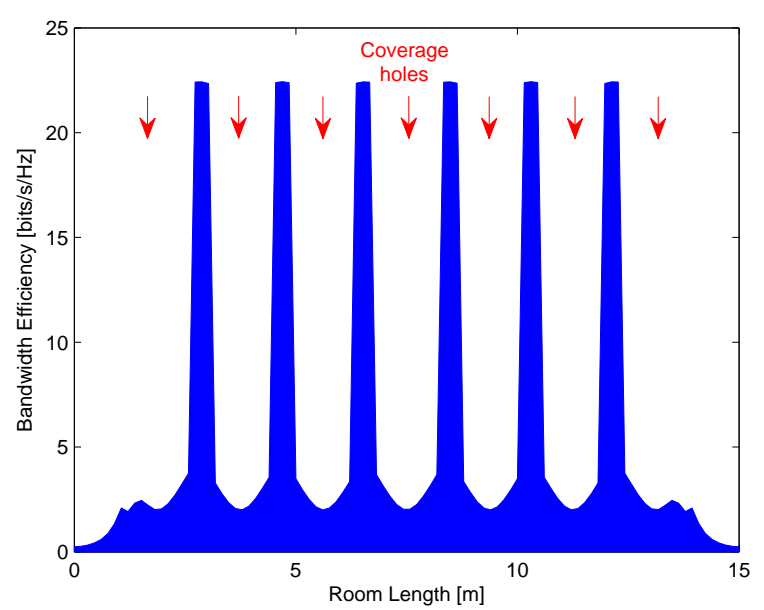

(b) UFR

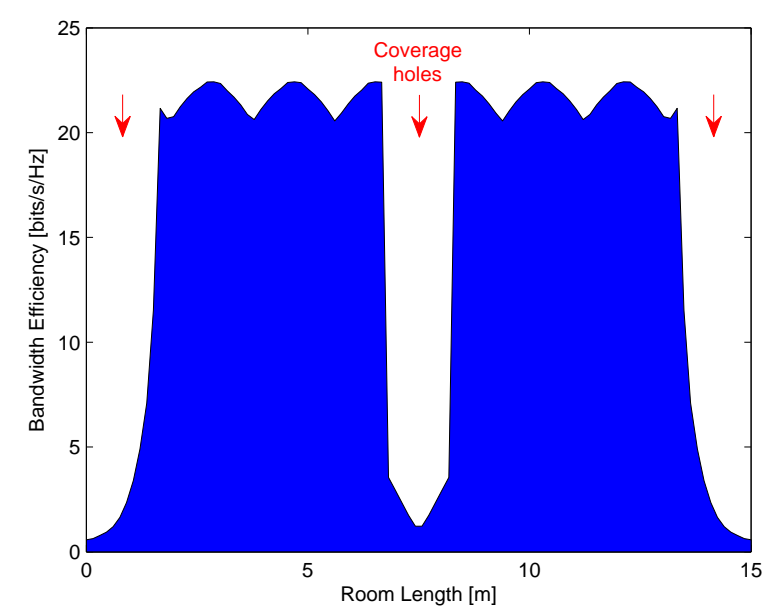

(d) VT-16

Fig. 9: (a) The ASE of different cell formations corresponding to the simulation parameters in Table IV. (b)-(d) The communication coverage holes in the UFR, FR-2 and VT-16 schemes, which are the cross section of Figure 8a, $8 \mathrm{~b}$ and 8 f, respectively.

statistics. A practical approach is to place the optical APs on a hexagonal or a square lattice [2]. For conventional RF base stations, the so-called Manhattan model based on a regular grid has been used, but it may be too idealized and inaccurate for the heterogeneous and ad hoc deployments, where the cell radii vary considerably due to differences in transmission power, tower height and user density [93]. Hence, another widely-used approach is to model the location of the RF base stations as Poisson point process [93]. Following the work in [93], Chen et al. [92] derived the analytical expressions for the SINR statistics of DCO-OFDM-aided VLC networks by employing the hexagonal and the Poisson point process based random AP distribution. As also suggested in [92], both of the above-mentioned two extreme cell deployments would be impractical, since they may require extra engineering work to redesign the lighting infrastructure. Therefore, the models adopted from RF cellular networks may be used to provide an upper and lower bound, respectively, for the SINR performance of other potential optical cell deployments in practice. Our analysis in this paper relies on a fixed optical AP deployment, which can be readily employed when considering different optical AP arrangements.

\section{B. User-Centric Cluster Formation}

Against the above-mentioned network-centric design philosophy, a UC cluster formation regime is proposed and studied in [22], [89]-[91], [94]-[96], as a beneficial counterpart of the existing network-centric cells. By definition, UC design is different from the network-centric design, where the network configuration is fixed, regardless of the tele-traffic. To elaborate a little further, the user-centric cluster formation in VLC networks may be reminiscent of the user-centric base station clustering technique for achieving coordinated multipoint transmission in conventional RF networks. For example, the user-centric design philosophy has been employed to form the radio-remote-head/user cluster for supporting the cloud 


\begin{tabular}{|c|c|}
\hline$\overline{\text { Year }}$ & ICI techniques \\
\hline 2010 & $\begin{array}{l}\text { Prince and Little [85] investigated the advanced cooperative } \\
\text { transmission regime of multiple LEDs relying on the time-of- } \\
\text { arrival synchronization. }\end{array}$ \\
\hline \multirow{3}{*}{2012} & $\begin{array}{l}\text { Wang et al. [78] proposed a novel VLC AP arrangement for } \\
\text { reducing the SNR variance. }\end{array}$ \\
\hline & $\begin{array}{l}\text { Ghimire and Haas [82] deployed a self-organizing allocation } \\
\text { mechanism for dynamically reuse the resources in an aircraft } \\
\text { cabin. }\end{array}$ \\
\hline & $\begin{array}{l}\text { Wu et al. employed OOK, PPM and PWM techniques in the } \\
\text { neighbouring optical cells, in order to achieve a high data rate } \\
\text { with seamless coverage. }\end{array}$ \\
\hline \multirow[t]{2}{*}{2013} & $\begin{array}{l}\text { Stefan and Haas [79] formulated and solved the optimization } \\
\text { problem of the LED-lamp placement. }\end{array}$ \\
\hline & $\begin{array}{l}\text { Guan et al. [80] investigated two LED deployment schemes } \\
\text { by using the classic swarm optimization algorithm, in order to } \\
\text { minimise the average outage area. }\end{array}$ \\
\hline 2014 & $\begin{array}{l}\text { Sung et al. [83] proposed a scalable OFDMA-based VLC } \\
\text { system. }\end{array}$ \\
\hline \multirow[t]{2}{*}{2015} & $\begin{array}{l}\text { Li et al. [76] investigated various VLC cell formations in a } \\
\text { hybrid VLC/WiFi system. }\end{array}$ \\
\hline & $\begin{array}{l}\text { Chen } \text { et al. [84] designed a pair of fractional FR schemes for } \\
\text { the DCO-OFDM-based optical attocell-based networks. }\end{array}$ \\
\hline \multirow{3}{*}{2016} & $\begin{array}{l}\text { Li et al. [89] proposed the UC cluster formation for interfer- } \\
\text { ence mitigation in VLC networks. }\end{array}$ \\
\hline & $\begin{array}{l}\text { Zhang et al. [90] designed an energy-efficient VLC system } \\
\text { relying on the amorphous UC cluster formation. }\end{array}$ \\
\hline & $\begin{array}{l}\text { Li } \text { et al. [91] designed an energy-efficient UC-VLC system for } \\
\text { scalable video streaming. }\end{array}$ \\
\hline
\end{tabular}

TABLE VIII: A brief summary of ICI mitigation schemes in VLC systems.

\begin{tabular}{|l|l|l|}
\hline \multicolumn{1}{|c|}{ Scheme } & \multicolumn{1}{|c|}{ Advantages } & \multicolumn{1}{|c|}{ Disadvantages } \\
\hline AP arrangement & $\begin{array}{l}\text { Simple implemen- } \\
\text { tation }\end{array}$ & $\begin{array}{l}\text { Subject to specific } \\
\text { scenarios }\end{array}$ \\
\hline Frequency planning & $\begin{array}{l}\text { Increased SINR } \\
\text { for cell-edge } \\
\text { users, simple } \\
\text { implementation }\end{array}$ & $\begin{array}{l}\text { Low bandwidth ef- } \\
\text { ficiency }\end{array}$ \\
\hline Cell coordination & $\begin{array}{l}\text { Higher bandwidth } \\
\text { efficiency }\end{array}$ & $\begin{array}{l}\text { Increased signal } \\
\text { processing } \\
\text { complexity }\end{array}$ \\
\hline UC cluster formation & $\begin{array}{l}\text { Consider user } \\
\text { information, } \\
\text { adjustable to any } \\
\text { AP arrangement }\end{array}$ & $\begin{array}{l}\text { Need central con- } \\
\text { trol unit }\end{array}$ \\
\hline
\end{tabular}

TABLE IX: Advantages and disadvantages of different ICI mitigation shcemes reported in VLC systems.

radio access networks [97]-[102]. By contrast, the conventional network-centric cell structure may be interpreted as a less sophisticated clustering technique, where the network is partitioned into non-overlapping clusters. Hence the clusteredge users of the conventional network-centric philosophy suffer from high inter-cluster interference.

First the so-called combined transmission (CT) technique is employed in each UC cluster, where multiple APs jointly convey the same information mapped to the same visible carrier frequency, while serving a single UE at a time. Considering the example seen in Figure 12, each hollow square and multiple APs (sometimes a single AP) constitute a UC cluster. When multiple UEs can receive data from the same $\mathrm{AP}$, only one of them can be granted access for the sake of avoiding extra interference, as discussed in [95]. For example, the solid square on the bottom right of Figure 12 and the other two neighbouring hollow squares can receive information from

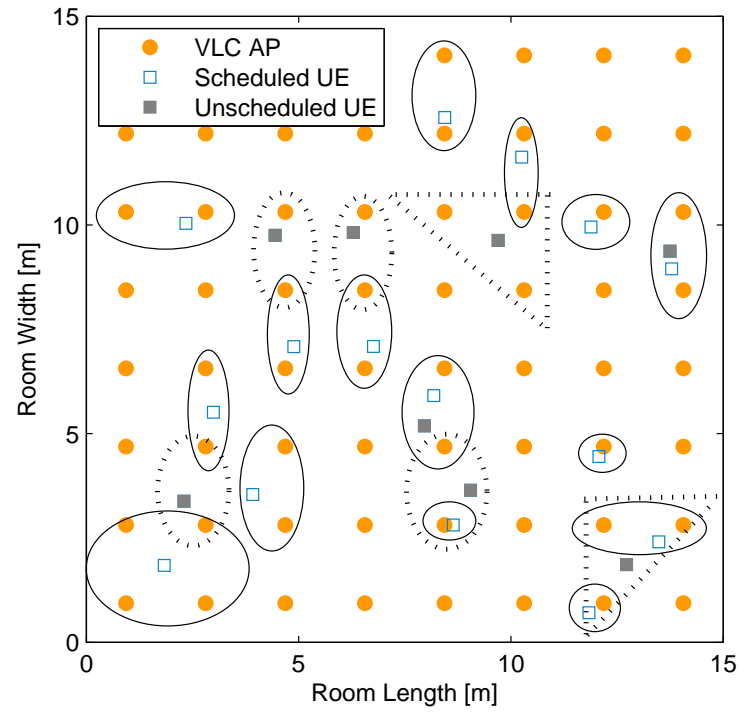

Fig. 10: The amorphous UC multi-AP clusters constructed for jointly transmitting data to a single UE by employing $\mathrm{CT}$, where the multiple APs convey the same information on the same visible carrier frequency and served a single UE at a time. When multiple UEs can receive data from the same AP, only one of them can be granted access for the sake of avoiding extra interference.

the same APs, while the solid square is not scheduled during the current slot. Its potential cluster is surrounded by a dashed ellipse. All the disjoint groups seen in Figure 12 are scheduled in a parallel manner upon using the scheduling algorithm of [95].

In order to further improve the bandwidth efficiency and serve multiple UEs at the same time, the so called vectored transmission (VT)-aided UC cluster formation philosophy was proposed in [22], [89], where each user-centric-vectoredtransmission-aided (UC-VT) cluster is served by a set of VLC APs, which simultaneously serve multiple UEs by employing VT. An example of two APs serving two UEs by using VT will be shown and discussed in the context of Figure 7d. More explicitly, a UC-VT cluster includes a set of APs and UEs as well as the transmission links between them. The concept of cluster formation is different from the concept of cell formation. Explicitly, the former may be defined as forming a UC cluster, where a set of VLC APs simultaneously serve multiple users by employing the advanced VT scheme of [76]. However, the concept of cell formation is more conventional, which may be defined as combining several optical APs to form a large multi-AP cell and to transmit data cooperatively, as shown in Figure 7c.

Figure 11 portrays the conventional cell formation structure (left) and the amorphous UC cluster formation (right) for a $15 \mathrm{~m} \times 15 \mathrm{~m}$ indoor VLC system having $8 \times 8$ APs (marked by squares) and 20 UEs (marked by circles) under three typical scenarios, where the UEs' positions are drawn from a uniform random distribution. Conventional cells typically have a fixed shape. For example, we may partition the $15 \mathrm{~m} \times 15 \mathrm{~m}$ 


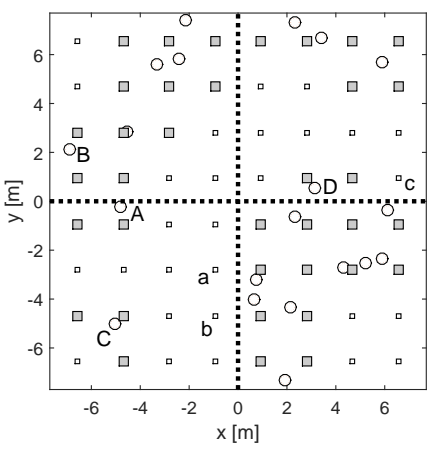

(a) Four network-centric cells

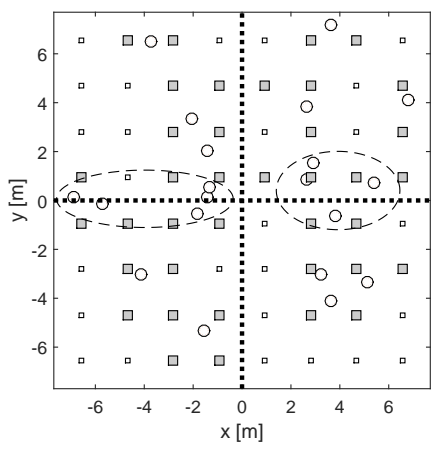

(c) Four network-centric cells

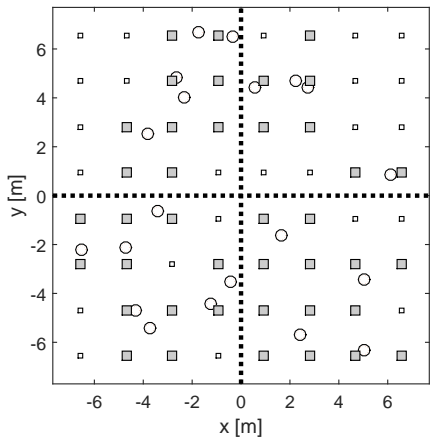

(e) Four network-centric cells

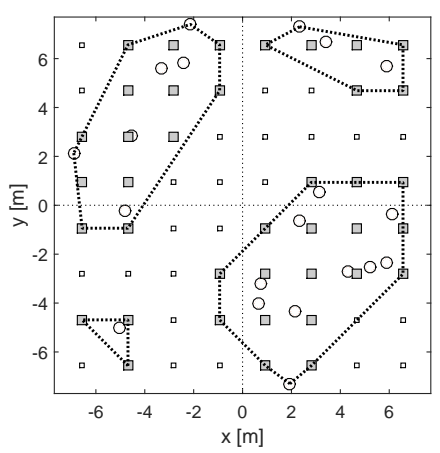

(b) Four UC clusters

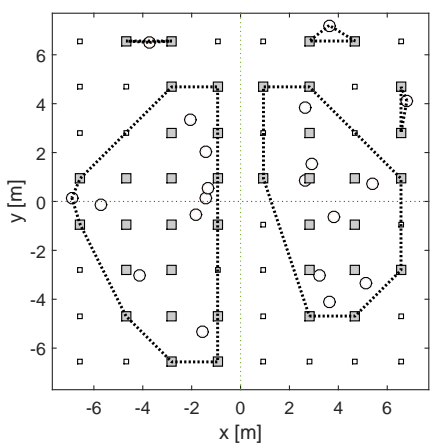

(d) Five UC clusters

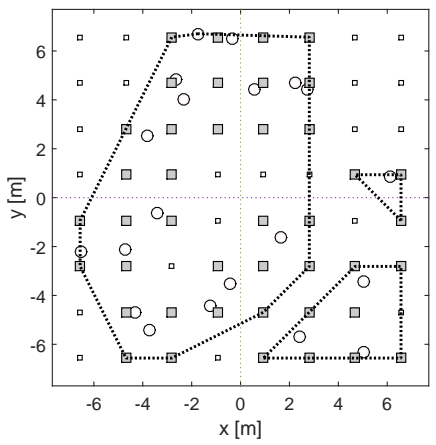

(f) Three UC clusters
Fig. 11: Illustration of the conventional (left) and the amorphous structure (right) for VLC indoor systems [90].

indoor environment into four square-shaped cells having $(4 \times 4)$ $=16 \mathrm{APs}$ per cell, where the users are associated with cells depending on the UEs' positions relative to the squareshaped boundary amongst the cells. We may switch off the communications function of the specific APs having no LoS links to the users in their vicinity (indicated by hollow small squares), in order to improve the energy efficiency. The related examples may be seen in the left of Figure 11. Note that the number of APs per cell may be pre-set as any feasible number.

Observe from the left subfigures of Figure 11 that this arrangement may not be the most appropriate one. For example, in the bottom-left corner of cell of Figure 11a, the 'boundary UE' ' $\mathrm{A}$ ' is clearly far from UE ' $\mathrm{C}$ ' in the same cell, but it is more close to UE ' $\mathrm{B}$ ' in the neighbouring cell. This is good reason for UE ' $\mathrm{A}$ ' to be separated from user ' $\mathrm{C}$ ' and to join UE 'B', as seen in Figure 11b of the distance-based UC cluster. This is also true for the 'boundary UE' ' $D$ ' in Figure 11a, since it is more close to the UEs located in the bottomright cell, than to the rest of the distant UEs in the same cell. More examples are shown in Figure 11c, where two clusters of boundary users - namely those highlighted by ellipses - join the UC clusters of Figure 11d. In addition to a different UEto-AP association, the status of APs is also different, where for example, APs ('a', 'b', 'c') were switched from idle mode in the conventional cells of Figure 11a to active mode in the UC clusters of Figure 11b, since they have LoS connections to the associated UEs. Hence, the proposed UC cluster formation is capable of rearranging the conventional boundaries, leading to five and three AP-UE clusters in Figure 11d and Figure 11f, whilst in Figure 11b there are four clusters. Hence, without a dynamic cell boundary, the UC cluster formation is updated to include the users moving in, arriving at or departing from the system, which leads to a flexible, "breathing" and evolving cell structure.

An example of the UC design principle is shown in Figure 12 [91]. The UEs' location information is assumed to be accurately acquired, which is also an ongoing research topic in the context of VLC networks [103]. Thus the mutual distances $d_{u, u^{\prime}}$ between any pair of UEs as well as the mutual distances $d_{a, u}$ between any AP-UE pair may be calculated. In order to control the size of the UC clusters, the distance constraints are pre-set as $d_{\mu}$ for the UEs and $d_{\alpha}$ for the APs within a single cluster. In other words, the distance between the UE and its cluster centre is no more than $d_{\mu}$ and the distance between the AP and its cluster centre is no more than $d_{\alpha}$. Let us now consider the UC cluster formation step by step.

Step 1) Initial UE selection: During this step, a UE is randomly selected as the starting point for constructing a tentative single-UE subset as part of the new cluster, as shown in Fig. 12a.

Step 2) Tentative UE-set expansion: The initial tentative UE set in Step 1 is expanded by including the nearby UEs within a certain range $d_{\mu}$, as seen in Fig. 12a. The centre of the tentative UE set should be updated by averaging the locations of all UEs in it, as shown in Fig. 12b. The expansion step should not stop until no more UEs are found in the proximity of the tentative UE subset.

Step 3) UE set formation: Following Step 2, the UE subset is deemed to be determined. Upon repeating Step 1 and 2, all the UE subsets have been constructed, as shown in Fig. 12c.

Step 4) Anchor AP selection: In order to guarantee that the number of APs is no less than the number of UEs within each UC cluster, each UE first finds its closest AP denoted by AP. If more than one UEs select the same anchor AP, this AP selects its closest UE and the other UEs have to select their next nearest APs, until each UE has successfully selected a unique anchor AP.

Step 5) Tentative AP-set adjustment: During this adjustment step, each tentative AP set is expanded first by including the nearby idle APs within a certain range $d_{\alpha}$. After gradually expanding all the tentative AP sets, if a specific AP was included in several tentative AP sets, then this AP is set to its idle mode, which is shown as the solid triangle in Fig. 12e. This measure is taken for the sake of avoiding any extra interference as well as for saving energy. Thus, after the 


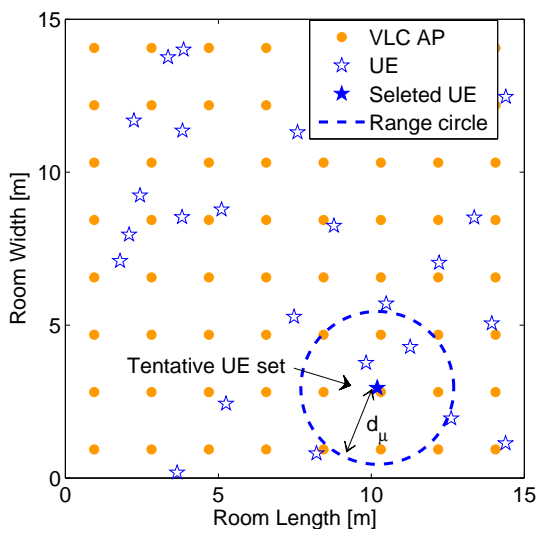

(a) Initial UE selection.

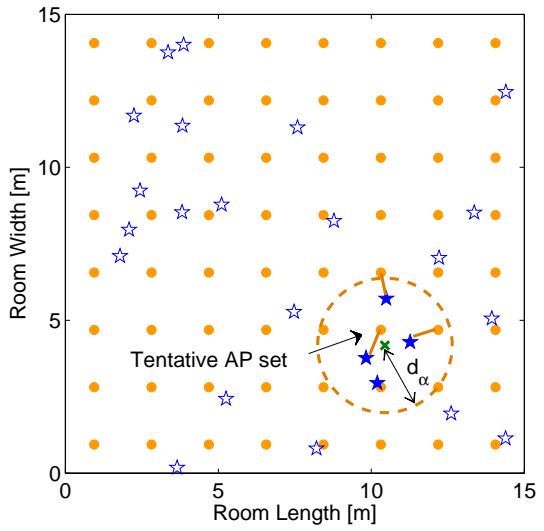

(d) Anchor AP selection.

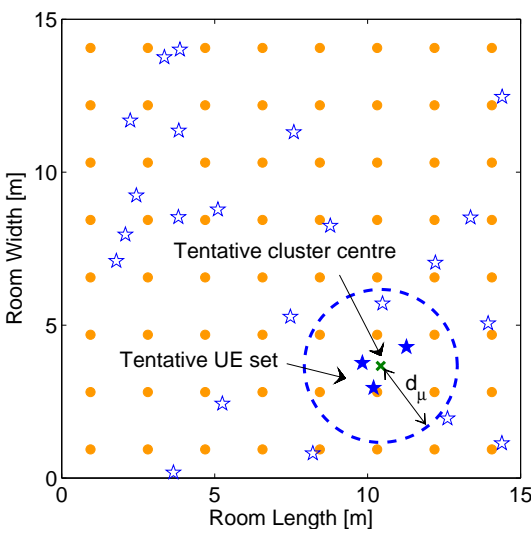

(b) Tentative UE-set expansion.

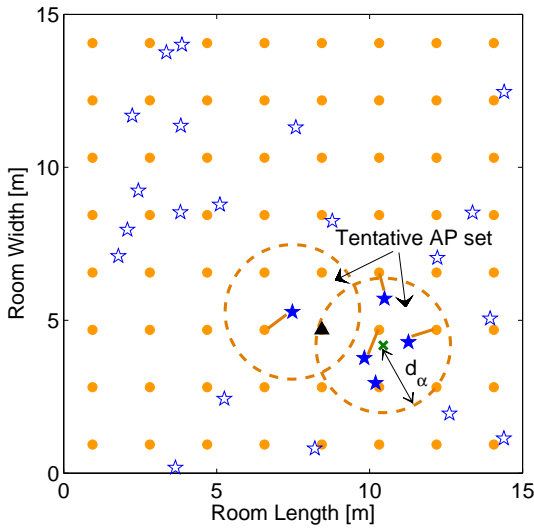

(e) Tentative AP-set adjustment.

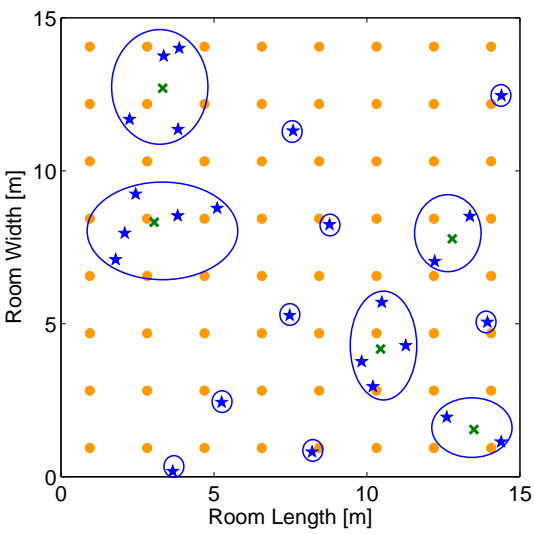

(c) UE-set formation.

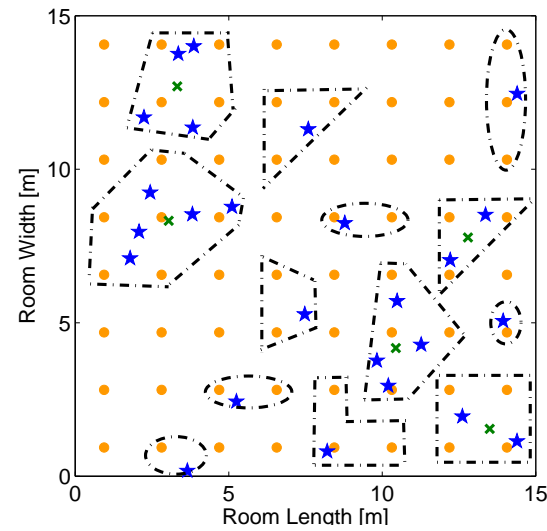

(f) UC cluster formation.

Fig. 12: Steps of UC cluster formation [91]. The subfigures (a)-(c) illustrate the formation of the UE set for a specific UC cluster. (d) and (e) show the formation of the AP set for an example UC cluster, where a UE is connected by a short line with its anchor AP. Note that these steps are carried out after all UE sets are formed. In (f), multiple UC clusters are finally constructed. Note that the area of the closed irregular shapes do not represent the coverage of each cluster and the inter-cluster interference is not illustrated.

process of exclusive assignment based expansion, all tentative AP subsets are determined.

Step 6) UC cluster formation: Upon combining the corresponding AP subset and UE subset, the UC clusters are finally constructed, as shown in Fig. 12f.

Following the novel UC design principle, Li et al. [89] studied a joint UC cluster formation and multiuser scheduling problem and proposed a low-complexity greedy algorithm for solving this essential issue in multiuser networks. On the other hand, [90] focused on the energy efficiency of the hitherto unexplored UC-VLC networks, despite the fact that most of the VLC research aimed for increasing the attainable throughput. Furthermore, the application of the UCVLC networks was further explored in [91], [96]. Explicitly, a combined triangulation-based and fingerprinting-based positioning technique was proposed for UC-VLC networks in [96], which is capable of achieving a high positioning accuracy at an appealingly low complexity. In [91], an energy-efficient indoor VLC system relying on dynamic UC cluster formation was designed for scalable video streaming, in order to further realize the energy efficiency potential of VLC.

\section{Multiuser Signal Processing}

In order to support multiple UEs simultaneously, multiuser signal processing techniques have to be invoked for UC cluster formation. Let us hence embark on a brief review of the multiuser signal processing techniques proposed for VLC systems.

Multiple input multiple output (MIMO) systems are capable of offering an increased data rate compared to the single input single output (SISO) systems. Hence, the research of advanced MIMO transmission techniques has also been intensified in the optical domain [103]-[115]. In particular, the authors of [104] and [105] investigated the free-space optical MIMO systems equipped with multiple lasers and PDs, while the treatises [106]-[110] are based on VLC systems. Explicitly, Zeng et al. [106] studied and compared the non-imaging and 
imaging MIMO techniques ${ }^{5}$, which provided deep insights concerning optical MIMO systems. An experimental $2 \times 2$ imaging-based MIMO system was reported in [107], but the illumination requirements were not given any cognisance. In addition, a MIMO system using an imaging receiver relying on a hemispherical lens was designed and analysed in [108] for improving the spatial diversity order. Relying on the wide FoV achieved by using the hemispherical lens, the optical signals received are effectively separated. Hence, the correlations between the elements of the channel matrix are relatively low, which provides a beneficial spatial diversity gain for the efficient decoding of the signal in a MIMO system. Furthermore, Azhar et al. [109] were the first authors, who demonstrated a MIMO-OFDM-based imaging receiver, which achieved a transmission rate of $220 \mathrm{Mbits} / \mathrm{s}$. Then they improved the attainable system performance by optimizing the pre-equalization weight of each carrier, where a transmission rate of $1 \mathrm{~Gb} / \mathrm{s}$ was achieved experimentally by a MIMO system, as reported in [110].

In contrast to the above single-user MIMO systems, the family of multiuser multiple-input-single-output (MU-MISO) techniques designed for VLC systems has also attracted much attention [103], [111]-[115]. In [111], a transmit precoding and biasing scheme was designed for the transmitter of a MUMISO broadcast system, where both the classic linear ZF and ZF dirty paper coding techniques were applied and evaluated. In addition, Li et al. [112] designed the optimal MU-MISO transceiver for VLC systems relying on the objective function of minimizing the maximum mean square error between the legitimate transmitted and received signals of the users. They have also considered the unique constraints of VLC systems, such as the real-valued nonnegative nature of the optical signal, the maximum tolerable optical intensity, and the constant brightness requirements of the LEDs. Recently, Shen et al. [115] optimized the achievable data rate of the VLC DL in a ZF-beamforming-aided MU-MISO system.

Furthermore, Biagi et al. [113] proposed a hybrid localization, access and transmission (LAT) solution for VLC networks by employing the so-called MIMO-LED scheme and the PPM modulation, which was capable of increasing the transmission rate without additional signal degradation. As a further advance in [103], Biagi et al. extended the LAT scheme to the localizing, accessing, scheduling and transmitting (LAST) scheme for their MIMO-VLC system and improved the data rate, when the number of users was increased. This was achieved by adaptively allocating communication frequency bands to multiple users and hence attaining a beneficial diversity gain in the frequency domain. Furthermore, Wang et al. [114] investigated the multiuser MIMO-OFDM (MU-MIMOOFDM) based VLC system, where the multiuser interference

\footnotetext{
${ }^{5}$ In a conventional non-imaging MIMO system, each of the multiple LED transmitters conveys an independent data stream simultaneously and an individual non-imaging optical concentrator is used by each receiver, where the desired signal, delayed multipath components, ambient light noise, and cochannel interference are combined into a single electrical signal. By contrast, in an imaging MIMO system, light propagates from each LED as before, but is projected onto a detector array [116]. The projected images may activate a single pixel or a group of pixels on the array, where each pixel of the detector array represents a receiver [106].
}

was eliminated by designing a precoding matrix individually handling each OFDM subcarrier. Different optical-OFDM schemes, namely the DCO-OFDM scheme associated with the minimum DC bias and the unified DC bias as well as the ACOOFDM scheme, were also studied and compared in [114].

\section{Multiuser Scheduling and Resource Management}

When multiple UEs are present in the VLC system, fair and efficient multiuser scheduling (MUS) and resource management constitutes one of the salient problems, which in fact affects all multiuser networks. The problem has been lavishly studied in the context of RF networks [117]-[120], but in VLC-based networks the problem has remained to a large extent hitherto unexplored in the open literature. Nonetheless, recently some valuable studies were disseminated in the context of network-centric single-AP VLC cells [103], [115], [121]-[127].

In particular, Bykhovsky and Arnon [121] proposed a heuristic scheme for beneficially allocating sub-carriers in a VLC multiple access system relying on DMT modulation, in order to improve the aggregate throughput. Again, the logical frame work referred to as LAST and proposed by Biagi et al. for VLC systems was capable of achieving a substantial throughput at a modest complexity. However, similar to most of the literature studying resource allocation in VLCbased systems, both [103] and [121] endeavoured to improve the attainable throughput without giving any cognisance to the fairness experienced by the UEs. By taking fairness into account, Huang et al. [122] proposed an incremental scheduling scheme, where the global scheduling phase is responsible for assigning the resources to the UEs, while the local scheduling phase regularly adjusts the resource allocation by tracking the UEs' movements. Since the VLC APs are sending beacon frames periodically, the users can send a response message whether they have received the beacons. Then the scheduler can find the moving users according to the feedback information. Furthermore, Babatundi et al. [123] proposed a proportional fairness based scheduling algorithm for a centrally controlled VLC system, which outperformed the maximum-rate scheduling policy in terms of balancing the achievable throughput against the fairness experienced by the UEs.

Additionally, compared to RF communications, VLC has some unique features, which should be carefully treated. Specifically, blocking is one of the most significant physical characteristics of VLC. Kim et al. [124] proposed three resource-allocation-based service modes for the VLC DL, in order to maintain both a high system throughput as well as satisfying the lighting requirements. Moreover, considering the nonnegativity of the intensity-modulated signals as another distinguishing feature of VLC, Park et al. [125] designed an optical MIMO system and proposed an optical power allocation scheme for the sake of maximizing the system's spectral efficiency. Furthermore, the non-orthogonal multiple access (NOMA) concept was also introduced into the VLC DL in order to increase the achievable throughput [126], where a novel power allocation strategy relying on the users' channel 
conditions was also proposed. According to [126], both the specific transmission angles of the LEDs and the receiver's particular FoV may be beneficially exploited to boost the attainable NOMA performance. As a further advance, another basic characteristic of the LEDs related to the particular colour perceived by the human eye, namely the chromaticity, was considered by Jiang et al. [127] as one of the constraints imposed on the sum-rate maximization problem, along with the constraints on the luminance, signal amplitude as well as on the target BER. Explicitly, in order to maximize the sum-rate of their MU-MISO VLC system relying on multichip LEDs, an electrical and optical power allocation scheme subject to the above-mentioned four constraints was proposed and evaluated [127].

\section{Applications AND Extensions of VLC}

As a promising complimentary extension to the wellestablished RF networks, VLC is becoming a promising enabler for providing indoor coverage, owing to its energyefficient nature, whilst simultaneously supporting both communications and illumination. In this section, we focus our attention on the application of VLC-based systems, including VLC-based HetNets, video streaming as well as its broader extension to the IoT.

\section{A. VLC-aided Heterogeneous Networks}

In order to support the rapidly increasing demand improved wireless data rates, as shown in Figure 1, both industry and academia are dedicated to seeking effective solutions, since we are practically approaching the theoretical limit of the RF channel capacity [128]. Exploiting new spectral bands may be expected to be one of the radical solutions, such as for example the millimeter-wave [4] and the optical wireless/VLC band [1]. In this paper, we focus our attention on the family of VLC systems, which is deemed to be a promising complementary extension to the well-established indoor RF networks. On the other hand, a stand-alone VLC system may exhibit several potential disadvantages, such as the lack of up-link transmission, poor performance in NLoS scenarios and small coverage for a single VLC AP. Hence, its RF counterpart may be invoked as a cooperative 'partner' in order to realize seamless communications. Although this integration may be an effective solution for satisfying the increasing throughput demand, there are some open challenges, such as for example the conception of cooperative load balancing and of efficient handovers.

1) Load Balancing: The concept of load balancing (LB) is interpreted in [129] as matching the demand for resources ('load') with the supply of resources ('capacity'), which constitutes one of the fundamental problems in many fields of engineering, logistics and economics. Moreover, the LB problem also affects all cooperative multi-rate HetNets. Substantial related work has been undertaken based on the LB problem in RF networks [3], [130]-[136]. In particular, Andrews et al. [129] investigated diverse technical approaches to the LB problems of HetNes and furthermore provided valuable design guidelines for OFDMA-based cellular systems. As also suggested in [129], the primary approaches included centralized optimization, game theory, Markov decision processes and the family of cell range expansion techniques.

Broadly speaking, the LB problem can be formulated as the constrained optimization of a carefully selected utility function [137], while satisfying the users' quality of service (QoS) requirements, which may be written as the generic optimization problem formulated below:

$$
\begin{array}{cl}
\max _{\boldsymbol{x}} & U_{0}(\boldsymbol{x}) \\
\text { s.t. } & f_{i}^{c}(\boldsymbol{x}) \leq C_{i} \quad 1 \leq i \leq m, \\
& f_{j}^{t}(\boldsymbol{x}) \geq T_{j} \quad 1 \leq j \leq k
\end{array}
$$

where $\boldsymbol{x} \in \mathbb{R}^{n}$ is the independent variable and $U_{0}$ is the selected utility function. Furthermore, $f_{i}^{c}$ is the $i$ th cost function and $C_{i}$ is the corresponding $i$ th $\mathrm{AP} /$ network resource limit, while $f_{j}^{t}$ denotes the $j$ th UEs achievable data throughput and $T_{j}$ corresponds to its throughput requirement. If (14)-(16) are all convex ${ }^{6}$, the problem is a convex optimization problem [137]. However, due to the coupled relationship between the user association and scheduling, the problem of (14)-(16) is usually NP-hard and may not be directly computable in a centralized manner. Since the subject of convex optimization has been widely studied [138], [139], a straightforward way is to relax the optimization problem (14)-(16) and make it convex. For example, with the aid of the classic dual decomposition approach, Ismail and Zhuang [135] developed a distributed resource allocation algorithm for the UEs associated with multi-homing capability in HetNets. Furthermore, by using the dual decomposition approach, Ye et al. [136] proposed a low-complexity near-optimal algorithm for the essential user association and LB problem after relaxing the binary variable.

The study of the LB problem in VLC-aided HetNets has intensified in recent years [76], [77], [140]-[144]. Specifically, in order to achieve accurate load balancing in sophisticated three-tier HetNets, which were constituted by the RF macrocells and femtocells as well as by the indoor optical attocells, Stefan and Haas [140] proposed a minimum-distance-based cell association scheme and showed that the average area spectral efficiency of the VLC-based HetNets was significantly increased compared to that of the stand-alone RF networks. Furthermore, the above-mentioned classic dual decomposition approach has also been invoked for solving the LB problems in VLC-aided HetNets [76], [77], [141]. Explicitly, Jin et al. [141] proposed a resource allocation scheme for a hybrid VLC/femtocell network under delay requirements and evaluated it by considering various unique characteristics of the VLC-based system. Furthermore, a cooperative LB solution was proposed for a hybrid VLC/WiFi system by $\mathrm{Li}$ et al. [76], where different VLC cell formations were considered. As a further advance, Wang and Haas [77] considered the users' mobility and proposed a dynamic LB scheme for hybrid VLC/RF HetNets.

\footnotetext{
${ }^{6} \mathrm{~A}$ function $f: \mathbb{R}^{n} \rightarrow \mathbb{R}$ is convex if, $\forall \boldsymbol{x}, \forall \boldsymbol{y} \in \operatorname{dom} f$ and $\theta \in[0,1]$, $\theta \boldsymbol{x}+(1-\theta) \boldsymbol{y} \in \operatorname{dom} f$ (i.e. the domain is a convex set) and $f(\theta \boldsymbol{x}+(1-\theta) \boldsymbol{y})$ $\leq \theta f(\boldsymbol{x})+(1-\theta) f(\boldsymbol{y})$.
} 
2) Efficient Handover: When considering a mobile user coming in a hybrid VLC/RF network, it may experience a high QoS in the centre of the VLC AP, while the QoS will be dramatically degraded, when it moves to the cell edge or when the LoS transmission is blocked. In this scenario, vertical handover (VHO) to the over-sailing RF cell should be invoked to maintain a smooth service quality. On the other hand, a VHO also occurs, when a RF user receives a stronger VLC signal and thus changes its connection from RF to VLC. Apart from the VHO, when the VLC APs are densely deployed, a user may experience several horizontal handovers between different VLC APs within a few dozen of metres.

Similarly to the existing RF-based HetNets, there are some potential problems, when invoking handovers in hybrid RF/VLC networks. Let us consider a user switching its connection from VLC to RF. When a new user arrives, the load of the RF AP is increased and the resources should be reallocated. Consequently, some of the existing users served by this RF AP only granted a reduced data throughput, while others may be assigned to the VLC network or to another RF AP. Conversely, in the specific VLC AP, where a user terminates his/her session, more resources become available for enhancing the QoS of its users. This is referred to as the knock-on handover effect [145]. Another problem is caused by transient LoS link blockages, which results in the user switching back and forth between the RF and VLC networks, hence increasing the system's signalling overhead. This is the so-called ping-pong effect [146]. In order to mitigate the adverse effects of frequent handovers, efficient handover techniques are essential for the success of VLC-based HetNets.

Although there is plenty of valuable research on handovers in RF networks [146]-[148], handover techniques conveived for VLC-based HetNets have remained to a large extent hitherto unexplored. In [149], the horizontal handovers between multiple VLC APs were studied in both non-overlapping spotlighting and in overlapping uniform lighting scenarios. Rahaim et al. [150] investigated the VHO criteria of a hybrid $\mathrm{VLC} /$ wireless fidelity (WiFi) network in order to improve both the service quality and the total throughput. As a further development, Liang et al. [151] proposed an advanced VHO algorithm relying on the prediction of transfer delay in a hybrid VLC/LTE system, which may be determined by service interruption duration, message size, access delay, etc. Wang and Haas [77] optimized the LB by taking into account the handover signalling overhead of a hybrid VLC/WiFi system. Furthermore, the classic decision-making algorithms, such as the fuzzy-logic decision making approach [152] as well as the Markov decision process [153], were also introduced into VLC-based HetNets for designing effective handover schemes. In particular, Hou and O'Brien [152] proposed a fuzzy-logicbased VHO algorithm by jointly considering a pair of VHO techniques. Specifically, for their immediate handover scheme, there is no dwell time and the VHO is immediately triggered when the optical channel is interrupted. By contrast, for their dwell-based handover scheme, the dwell time is set to the maximum tolerable duration of a short service interruption, after which the VHO will be triggered. Moreover, Wang et al. [153] formulated the VHO problem as a Markov decision process and solved it in a dynamic manner in a VLC/RF system at a low signalling cost without any obvious system performance degradation.

\section{B. Video Streaming}

According to the global mobile data traffic statistics recently released by Cisco [7], video streaming is and will continue to be the most dominant form of tele-traffic, which will account for three quarters of all tele-traffic in 2021, as seen in Figure 1. As one of the 'killer'-applications in contemporary wireless communications systems [154]-[156], video streaming has become a popular research topic, including efficient compression standards [157]-[159], error-concealment/error-resilient streaming techniques [155], [160], [161], as well as reliable transmission schemes [162]-[164], etc.

1) Conventional Video Coding: Owing to its high data rate potential and its inherent energy efficiency, VLC may be expected to become an additional promising enabler for video transmission. Insightful research has been dedicated to video streaming in VLC-based systems [156], [165]-[168]. Explicitly, Vučić et al. introduced a VLC prototype, which successfully transmitted three high-definition video streams in a parallel manner, while Rufo et al. [166] characterized the impact of specific illumination levels on their video transmission scheme relying on their VLC prototype system. A VLC system achieving a video transmission rate of 10 $\mathrm{Mb} / \mathrm{s}$ over a short link was presented by Chen et al. [167]. Furthermore, a VLC-based audio/video system, which was capable of providing real-time interaction and simultaneous availability of both video and audio was reported in [168]. In order to minimize the video distortion, a novel multiuser video streaming optimization framework was conceived for efficient video delivery by Jiang et al. [156], while considering various VLC cell formations. Finally, they also provided design guidelines for improving the overall video quality of VLC-based video streaming systems.

2) Scalable Video Coding: In order to meet the requirements of the various transmission scenarios in the era of the Internet and of mobile networks, the advanced scalable extension of the so-called high-efficiency video coding (SHVC) techniques [159] has emerged and gained popularity, as a benefit of its scalable nature. Explicitly, this scheme is capable of offering diverse visual qualities by promptly adapting to the time-variant channel conditions of different UEs. The SHVCbased layered video stream is constituted by multiple unequalimportance layers, which are generated by using carefully designed source codes [169], [170] as well as adaptive modulation and channel coding schemes [171], [172]. According to [173], if a carefully constructed subsets of the original video sequence may lead to video reconstruction either at a reduced picture size or at a reduced frame rate compared to the original one, then this video scheme is deemed to exhibit either spatial or temporal scalability, respectively. Another popular scalability mode is the so-called quality-scalability, where the subsets of bits may provide a reduced video fidelity. Explicitly, the fidelity is often represented in terms of the peak signal-tonoise ratio. As a further advance, the afore-mentioned modes 
of scalability may be supported by a single scalable video sequence.

The benefits of scalable video are multi-fold, amongst others, allowing for example the video decoder to progressively refine the reconstructed visual quality, as the channelquality improves. The hierarchical structure of the videostream also facilitates energy-efficient video communications by jointly considering the scalable video quality and the power consumption, as demonstrated for traditional RF networks in [174]-[176]. However, there is a paucity of studies on scalable video transmission in VLC-based systems [91], [177]. To fill this gap in the literature, Zhu et al. [177] conceived a CSK-aided unequal-error-protection scheme for layered video streaming in a VLC DL system, which outperformed the traditional equal error protection scheme. Moreover, $\mathrm{Li}$ et al. [91] further explored the energy efficiency of UC-VLC networks and designed an efficient scalable video system relying on dynamic UC cluster formation by jointly considering both the video-related and the VLC-related characteristics. Again, the transmission of scalable videos in VLC-based networks has remained to a large extent hitherto unexplored.

\section{Internet-of-Things}

HetNets relying on a diverse spectrum allocation constitute an integral part of the IoT, where VLC provides a vast supply of unregulated spectrum and plays an important role. Let us now discuss two significant applications of VLC techniques in IoT scenarios, namely in indoor positioning and vehicular communications.

Location-aware technologies will revolutionize many aspects of the commercial and public services, as well as the military sectors, and are expected to spawn numerous unforeseen applications [178]. Although the global positioning system (GPS)-based outdoor localization has been widely utilized, the accuracy of the indoor localization techniques may not be satisfactory. Compared to the metre-level accuracy of the outdoor localization, the error range of the indoor environment should be much lower, since the area of the indoor environment is usually limited to dozens of square metres. The straightforward approach is to use low-cost WiFibased indoor localization [179], but the achievable accuracy is low, on the order of 3-6 meters provided by the conventional WiFi-based localization approach. As a member of the indoor communication family, VLC is also expected to provide indoor localization services. It was shown in [180] that the number of LED lamps is usually more than that of the WiFi APs, which is a potential advantage of the triangulation based indoor localization technique. A practical VLC-based localization scheme was proposed in [180], where the localization accuracy is around $\sim 0.4 \mathrm{~m}$. Valuable research has also been undertaken related to VLC-based positioning in [96], [181]-[187].

Apart from the indoor localization applications, VLC techniques have also been employed in outdoor vehicle to vehicle $(\mathrm{V} 2 \mathrm{~V})$ and vehicle to infrastructure (V2I) communication scenarios [17], [188]-[195]. One of the most important applications of VLC-based vehicular communications is related to road traffic safety, where the traffic lights broadcast signals and approaching vehicles send hazard warnings. In these processes, the undesirable interference imposed by ambient light may not be negligible, as exemplified by street lights and the light emanating from the buildings. This problem was discussed in most of the relevant literature. On the other hand, the advantages of employing VLC for vehicular communications are multi-fold. Specifically, as a benefit of the self-positioning capability, the real-time information of neighboring vehicles is readily available for sharing and the drivers can adjust their driving conditions accordingly, which is capable of dramatically reducing the risk of collisions. Furthermore, LED lamps have already been routinely installed on vehicles, which reduces the extra cost of communications compared to deploying RF-based devices.

\section{Standarization and Commecialization}

As a viable commercial technology, the growing interest in the development of VLC is evidenced by the rich activities in academia, industry and standardization [196]. The Japan Electronics and Information Technology Industries Asociation (JEITA) standards of CP-1221 (Visible Light Communications System), CP-1222 (Visible Light ID System) CP-1223 (Visible Light Beacon System) were established by the Visible Light Communications Consortium (VLCC) in 2007, 2007 and 2013, respectively. The new standard International Electrotechnical Commission (IEC) 62943 (Visible Light Beacon System for Multimedia Applications) was published in March 2017, which is based on the JEITA CP-1223 standard and aims for establishing a unified standard concerning the lower communication layer common to multimedia applications.

By further considering the practical flckering and dimming issues, the IEEE published the 802.15.7 standard in 2011 [66] for providing a global standard for short-range optical wireless communication using visible light. This standard considers three classes of VLC devices, i.e. the infrastructure, mobile, and vehiclular device, and their features are listed in Table $\mathrm{X}$. Each VLC device is comprised of a PHY layer, which contains the light transceiver along with its low-level control mechanism and a medium access control (MAC) sublayer that provides access to the physical channel for all types of transfers. Three PHY layer types are defined with the data rate ranging from $11.67 \mathrm{~kb} / \mathrm{s}$ to $96 \mathrm{Mb} / \mathrm{s}$ and three MAC topologies are supported, i.e. star, peer-to-peer and broadcast topology. Although the IEEE 802.15.7 standard relies on diverse techniques, some of the latest technological developments in the field of VLC were not available when the standard was ratified. For example, OOFDM modulation methods have been widely studied and optimized for improving their data rates and energy efficiency [61]-[63], which may be the inspiration for a new VLC standard.

With the rapid development of the VLC techniques, the commercialization activities of VLC have also been intensified. In 2010, St. Cloud signed a contract with LVX Minnesota for LED lighting solutions and became the first to commercially deploy VLC services [197]. In July 2011, Prof. Harald Haas presented a live demonstration, where high-definition video was transmitted from a LED lamp, as shown at the 


\begin{tabular}{|l|l|l|l|}
\hline & Infrastructure & \multicolumn{1}{|c|}{ Mobile } & \multicolumn{1}{|c|}{ Vehicle } \\
\hline Fixed coordinator & Yes & No & No \\
\hline Power supply & Ample & Limited & Moderate \\
\hline Form factor & Uncostrained & Constrained & Uncostrained \\
\hline Light source & Intense & Weak & Intense \\
\hline Plysical mobility & No & Yes & Yes \\
\hline Range & Short/Long & Short & Long \\
\hline Data rates & High/Low & High & Low \\
\hline
\end{tabular}

TABLE X: Classification of VLC device in [66].

TEDGlobal [198] lecture. A promising application using VLC relies on indoor positioning [96]. In 2015, Philips collaborated with supermarket giant Carrefour to deliver VLC locationbased services to shoppers' smartphones in a supermarket in Lille, France [199]. However, the pervasive large-scale commercialization of VLC products will depend on the success of further engineering solutions as well as on their commercialization strategies [200].

\section{Design Guidelines ANd Open Challenges}

In this paper, we reviewed the characteristics of both pointto-point VLC transmission as well as of multiuser VLCaided networks, with a special emphasis on the radically new user-centric design philosophy. Compelling solutions to the salient problems of multiuser signal processing, scheduling and resource management have been surveyed. Furthermore, we also reviewed the applications of VLC systems as a valuable member of next-generation communication networks.

\section{A. Design Guidelines}

As explicitly shown in Figure 13, taking the entire community's design goals into consideration, we distilled the design guidelines listed below:

- Both illumination and communication requirements should be considered, when designing the deployment of optical APs, where multi-objective optimisation functions may be formulated and Pareto optimality may be achieved. More practically, VLC networks may employ the existing lighting infrastructure and efficient transmission as well as resource allocation schemes can be designed for it.

Remarks: As suggested in Section III-A4, the existing deployment model of RF base stations may also be considered for VLC networks on a smaller scale. Explicitly, the regular hexagonal/square grid arrangements are simple to implement and capable of offering uniform illumination. Compared to the hexagonal model, the square model may be more practical owing to its compliance with regular-shaped rooms. Furthermore, the widely-used Poisson point process based random RF network models may not constitute a practical approach for VLC systems, since the lighting infrastructure is seldom deployed in a random manner. In order to avoid having neighbouring APs extremely close to each other in a Poisson point process based network, the hard-core point process network studied in [92] may be considered as another practical solution for VLC AP deployment.
- From a network-centric perspective, according to Figure 8 and 9a, the FR regime is expected to be a simple and effective scheme conceived for reducing both the ICI and the coverage holes, when the throughput requirements are modest. If the desired throughput is increased, multi-AP cells employing VT schemes are recommended.

- For network-centric multi-AP cells, the specific number of APs within a single merged cell should be carefully selected, by considering both the desired QoS as well as the acceptable complexity.

- According to the investigations of [22], [89]-[91], [94][96], the user-centric design philosophy is capable of offering a superior performance over that of the conventional network-centric cell formations. Hence, we recommend the user-centric cluster formation for VLC systems.

- The integration of VLC and RF networks is essential, in order to achieve a seamless coverage, when the VLC DL cannot provide satisfactory QoS or if it is not available, for example owing to blocking. Hence, effective vertical handover schemes have to be used. Furthermore, since the up-link transmission may not be conveniently supported by stand-alone VLC systems, the up-link may have to rely on the classic RF network.

\section{B. Open Challenges}

1) Uplink: Since the uplink transmission from the mobile devices relying on VLC may be wasteful in terms of its energyconsuming and may impose visual disturbances, most of the open literature focuses on the VLC DL transmission [76]. In order to solve this uplink problem, both RF- [150] and infrared-based [166] techniques may be employed. Furthermore, WiFi has been ubiquitously rolled out in indoor environments, which is capable of providing convenient uplink transmissions for VLC-based HetNets. In the meantime, WiFi can also ensure a seamless coverage and an uninterrupted service, when the VLC channel is poor or blocked [77].

2) Back-haul: Apart from the up-link transmission, the provision of a high-speed, low delay back-haul is another important design issue in VLC-based HetNets. The popular approaches rely on power-line [201], [202] and on fibre, respectively. Since power-line communication (PLC) uses the existing power-lines and the ubiquitous wires for communication, it may be readily employed. PLC fits in very well with VLC, as the data in the power line can be used to modulate LEDs directly [203], [204]. The main challenges in PLC include high interference from appliances connected to the network, impedance and channel variations, non-white noise and phase changes [205]. It was suggests that a speed of 600 $\mathrm{Mb} / \mathrm{s}$ is possible over power lines [206]. In addition to MIMO solutions, relaying techniques were also proposed [207] for integrating PLC with VLC. More explicitly, various integrated VLC and power-line communication systems were proposed and investigated in [208]-[211]. An alternative approach is the fibre-based system, but given the high-density deployment of LEDs, the fibre-based infrastructure may be deemed expensive. Hence, the most appropriate design of the VLC back-haul remains a challenging open problem to explore. 


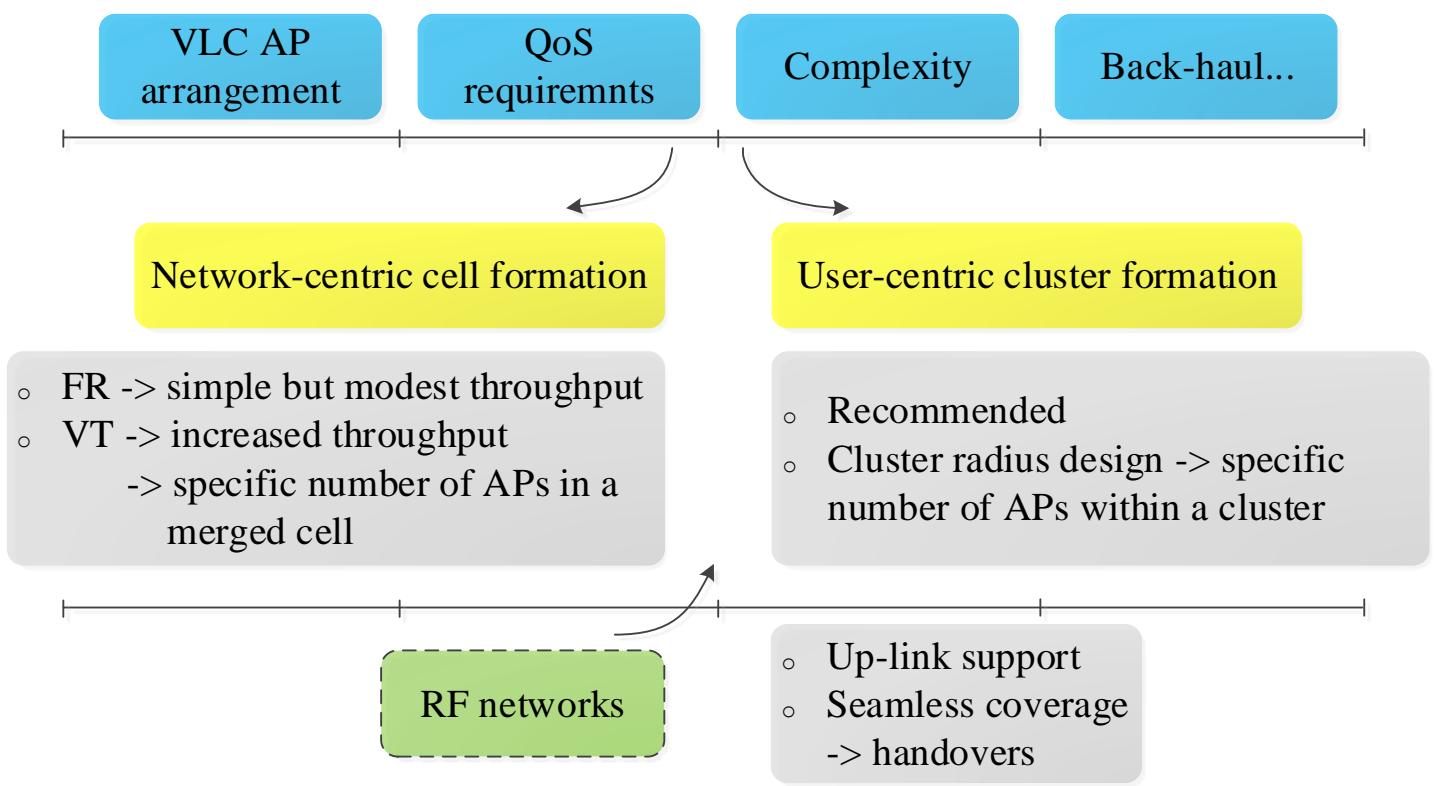

Fig. 13: Design guidelines for cell formations in VLC-aided systems.

3) Blockage: Due to the random blockage of LoS by the users' movement or owing to obstructing objects, the received optical power may be dramatically reduced and the performance of VLC is consequently degraded. Frequently switching between the VLC and cooperative RF networks may result in the so-called ping-pong effect, as stated in Section IV-A2. In order to mitigate the amount of control signalling and to reduce latency, a predictive handover decision should be adopted [212]. However, except for the preliminary study of the shadowing effects introduced in Section II-B3, the research of the related blockage issues remains limited in the literature. More explicitly, to capture and predict the blocking patterns caused by the users' random movements, a Kalman filter can be used to track the movement of the user and Markov chain based modelling can be invoked to predict the probability of LoS blocking. Then, based on this prediction, an improved access system association and resource allocation decision can be achieved.

4) Mobility: When the users are moving, mobility management constitutes another significant issue in VLC-based HetNets. Within a single VLC cell, the mobile user will experience substantially varying channel quality between the cell centre and the cell edge. This effect should be taken into consideration, when deploying the VLC APs as well as designing link-level techniques. Furthermore, the overlapping areas of neighbouring VLC APs may be contaminated by ICI, which dramatically degrades the QoS. Hence, the transmission schemes conceived for these ICI-infested areas should be carefully designed for ensuring the service remains uninterrupted. Although various FR techniques have been proposed, an inherent disadvantage is that switching between different optical frequencies every few meters degrades the user experience. Therefore, seamless horizontal handover and
VHO should be supported in VLC-based HetNets.

5) Outdoor Scenarios: In outdoor environments, data transmission mainly relies on LoS links in VLC systems, where the communication range is critically dependent on the intensity of the transmitted light. Impairments are imposed by many sourses, such as sunlight, street lights, etc., which may dramatically degrade the received signal. An optical filter may be invoked for mitigating the effects of the ambient light impairment by selectively transmitting the desired light wavelengths. Furthermore, the atmospheric conditions also affect the optical signal. Intuitively, the intensity of the light is reduced in fog and rain [213]. Hence, appropriate techniques should be designed for outdoor VLC systems, including their channel modelling [214], coding and modulation [215], detection [216], etc. Apart from the PHY-layer challenges, the handover problems also constitute an essential limitation of outdoor VLC systems, since vehicles are moving at a relatively high speed compared to the cell-radius [217].

6) LED Limitations: With the emergence of indium gallium nitride technology it has become possible to manufacture efficient white LEDs. For mass-production, the illumination industries still use large-area blue LEDs and phosphor colour converters, which however have a limited bandwidth of a few $\mathrm{MHz}$ and hence their transmission-rate remains limited. Although the high-bandwidth LEDs discussed in [20] are promising, they are still far from becoming the norm in the illumination industry. Another important issue in LEDs is their nonlinear current-power response and limited dynamic range, which results in both amplitude and phase distortions. The impact of nonlinearity is more severe for high-order sub-carrier modulation based OFDM due to its high peakto-average power ratio [20]. Hence, valuable research has been dedicated to treating the nonlinearity of LEDs, such as 
limiting the peak-to-peak input signal range [218], [219], using pre-distortion [220], post-distortion [221], [222] or nonlinear equalisers [223], etc. All in all, it is an exciting era for VLC researchers.

\section{REFERENCES}

[1] L. Hanzo, H. Haas, S. Imre, D. O’Brien, M. Rupp, and L. Gyongyosi, "Wireless myths, realities, and futures: From $3 \mathrm{G} / 4 \mathrm{G}$ to optical and quantum wireless," in Proceedings of the IEEE, vol. 100, May 2012, pp. $1853-1888$.

[2] A. Goldsmith, Wireless communications. Cambridge University Press, 2005.

[3] J. Andrews, S. Buzzi, W. Choi, S. Hanly, A. Lozano, A. Soong, and J. Zhang, "What will 5G be?" IEEE Journal on Selected Areas in Communications, vol. 32, no. 6, pp. 1065-1082, June 2014.

[4] S. Rangan, T. Rappaport, and E. Erkip, "Millimeter-wave cellular wireless networks: Potentials and challenges," Proceedings of the IEEE, vol. 102, no. 3, pp. 366-385, Mar. 2014.

[5] A. G. Bell, W. G. Adams, Tyndall, and W. H. Preece, "Discussion on the photophone and the conversion of radiant energy into sound," Journal of the Society of Telegraph Engineers, vol. 9, no. 34, pp. 375383,1880 .

[6] Y. Tanaka, S. Haruyama, and M. Nakagawa, "Wireless optical transmissions with white colored LED for wireless home links," in 2000 The 11th IEEE International Symposium on Personal, Indoor and Mobile Radio Communications, vol. 2, Sept. 2000, pp. 1325-1329.

[7] "Cisco visual networking index: Global mobile data traffic forecast update, 2016-2021 white paper," Feb. 2017. [Online]. Available: http://www.cisco.com/c/en/us/solutions/collateral/serviceprovider/visual-networking-index-vni/mobile-white-paper-c11520862.html

[8] Z. Ghassemlooy, W. Popoola, and S. Rajbhandari, Optical wireless communications: system and channel modelling with MATLAB. CRC Press, 2012.

[9] N. Kumar and N. R. Lourenco, "LED-based visible light communication system: a brief survey and investigation," Journal of Engineering and Applied Sciences, vol. 5, no. 4, pp. 296-307, Apr. 2010

[10] D. Tsonev, S. Videv, and H. Haas, "Light fidelity (Li-Fi): towards alloptical networking," in SPIE OPTO. International Society for Optics and Photonics, Feb. 2014, pp. 900 702-1-900 702-10.

[11] A. Sevincer, A. Bhattarai, M. Bilgi, M. Yuksel, and N. Pala, "LIGHTNETs: Smart LIGHTing and mobile optical wireless NETworks - a survey," IEEE Communications Surveys \& Tutorials, vol. 15, no. 4, pp. 1620-1641, Fourth Quarter 2013

[12] S. Wu, H. Wang, and C. Youn, "Visible light communications for 5G wireless networking systems: from fixed to mobile communications," IEEE Network, vol. 28, no. 6, pp. 41-45, Nov. 2014.

[13] D. Karunatilaka, F. Zafar, V. Kalavally, and R. Parthiban, "LED based indoor visible light communications: State of the art," IEEE Communications Surveys \& Tutorials, vol. 17, no. 3, pp. 1649-1678, Third Quarter 2015.

[14] P. H. Pathak, X. Feng, P. Hu, and P. Mohapatra, "Visible light communication, networking, and sensing: A survey, potential and challenges," IEEE Communications Surveys \& Tutorials, vol. 17, no. 4, pp. 20472077, Fourth Quarter 2015.

[15] N. Saha, M. S. Ifthekhar, N. T. Le, and Y. M. Jang, "Survey on optical camera communications: challenges and opportunities," IET Optoelectronics, vol. 9, no. 5, pp. 172-183, Oct. 2015.

[16] Y. Qiu, H. Chen, and W. Meng, "Channel modeling for visible ligh communications - a survey," Wireless Communications and Mobile Computing, vol. 16, no. 14, p. 2016?2034, Oct. 2016.

[17] A. M. Cailean and M. Dimian, "Toward environmental-adaptive visible light communications receivers for automotive applications: A review," IEEE Sensors Journal, vol. 16, no. 9, pp. 2803-2811, May 2016.

[18] K. Sindhubala and B. Vijayalakshmi, "Survey on noise sources and restrain techniques in visible-light communication," Light \& Engineering, vol. 24, no. 2, pp. 107-117, Apr. 2016.

[19] T. Do and M. Yoo, "An in-depth survey of visible light communication based positioning systems," Sensors, vol. 16, no. 5, p. 678, May 2016.

[20] S. Rajbhandari, J. J. D. McKendry, J. Herrnsdorf, H. Chun, G. Faulkner, H. Haas, I. M. Watson, D. O'Brien, and M. D. Dawson, "A review of gallium nitride leds for multi-gigabit-per-second visible light data communications," Semiconductor Science and Technology, vol. 32, no. 2, p. 023001, Jan. 2017.
[21] A. M. Cailean and M. Dimian, "Toward environmental-adaptive visible light communications receivers for automotive applications: A review," IEEE Sensors Journal, vol. 16, no. 9, pp. 2803-2811, May 2016.

[22] R. Zhang, J. Wang, Z. Wang, Z. Xu, C. Zhao, and L. Hanzo, "Visible light communications in heterogeneous networks: Paving the way for user-centric design," IEEE Wireless Communications, vol. 22, no. 2, pp. 8-16, Apr. 2015.

[23] C. W. Chow, C. H. Yeh, Y. F. Liu, and Y. Liu, "Improved modulation speed of LED visible light communication system integrated to main electricity network," Electronics Letters, vol. 47, no. 15, pp. 867-868, July 2011

[24] T. Komine, "Visible light wireless communications and its fundamental study," Ph.D. dissertation, Ph. D. dissertation, Keio University, 2005.

[25] Cree Inc., "Cree sets new R\&D performance record with 276 lumen-per-watt power LED," Feb. 2013. [Online]. Available: http://www.cree.com/News-and-Events/Cree-News/PressReleases/2013/February/276-LPW.

[26] J. Grubor, S. Randel, K.-D. Langer, and J. Walewski, "Broadband information broadcasting using LED-based interior lighting," Journal of Lightwave Technology, vol. 26, no. 24, pp. 3883-3892, Dec. 2008.

[27] D. A. Steigerwald, J. C. Bhat, D. Collins, R. M. Fletcher, M. O. Holcomb, M. J. Ludowise, P. S. Martin, and S. L. Rudaz, "Illumination with solid state lighting technology," IEEE Journal of Selected Topics in Quantum Electronics, vol. 8, no. 2, pp. 310-320, Mar. 2002.

[28] C. Yen-Chih, "Multi-chip LED," Jan. 2013, uS Patent D675,169.

[29] P. E. Burrows, G. Gu, V. Bulovic, Z. Shen, S. R. Forrest, and M. E. Thompson, "Achieving full-color organic light-emitting devices for lightweight, flat-panel displays," IEEE Transactions on Electron Devices, vol. 44, no. 8, pp. 1188-1203, Aug. 1997.

[30] J. J. D. McKendry, D. Massoubre, S. Zhang, B. R. Rae, R. P. Green, E. Gu, R. K. Henderson, A. E. Kelly, and M. D. Dawson, "Visible-light communications using a CMOS-controlled micro-light- emitting-diode array," Journal of Lightwave Technology, vol. 30, no. 1, pp. 61-67, Jan. 2012.

[31] T. Komine and M. Nakagawa, "Fundamental analysis for visiblelight communication system using LED lights," IEEE Transactions on Consumer Electronics, vol. 50, no. 1, pp. 100-107, Feb. 2004.

[32] A. Zukauskas, M. S. Shur, and R. Gaska, Introduction to solid-state lighting. J. Wiley, 2002

[33] "Cree XLamp XP-E high-efficiency white LEDs," 2015 [Online]. Available: http://www.cree.com/ /media/Files/Cree/LEDComponents-and-Modules/XLamp/Data-andBinning/XLampXPEHEW.pdf.

[34] G. Wyszecki and W. S. Stiles, Color science. Wiley New York, 1982.

[35] J. Kahn and J. Barry, "Wireless infrared communications," in Proceedings of the IEEE, vol. 85, no. 2, Feb. 1997, pp. 265-298.

[36] F. R. Gfeller and U. Bapst, "Wireless in-house data communication via diffuse infrared radiation," in Proceedings of the IEEE, vol. 67, no. 11, Nov. 1979, pp. 1474-1486.

[37] K. Lee, H. Park, and J. R. Barry, "Indoor channel characteristics for visible light communications," IEEE Communications Letters, vol. 15, no. 2, pp. 217-219, Feb. 2011.

[38] K. Lee and H. Park, "Channel model and modulation schemes for visible light communications," in 2011 IEEE 54th International Midwest Symposium on Circuits and Systems (MWSCAS), Aug. 2011, pp. 1-4.

[39] S. Rajbhandari, Z. Ghassemlooy, and M. Angelova, "Bit error performance of diffuse indoor optical wireless channel pulse position modulation system employing artificial neural networks for channel equalisation," IET Optoelectronics, vol. 3, pp. 169-179(10), Aug. 2009

[40] D.-q. Ding and X.-z. Ke, "A new indoor VLC channel model based on reflection," Optoelectronics Letters, vol. 6, no. 4, pp. 295-298, July 2010.

[41] J. B. Carruthers and J. M. Kahn, "Modeling of nondirected wireless infrared channels," IEEE Transactions on Communications, vol. 45 , no. 10 , pp. 1260-1268, Oct. 1997.

[42] V. Jungnickel, V. Pohl, S. Nonnig, and C. von Helmolt, "A physical model of the wireless infrared communication channel," IEEE Journal on Selected Areas in Communications, vol. 20, no. 3, pp. 631-640, Apr. 2002.

[43] L. Zeng, D. O’Brien, H. Le-Minh, K. Lee, D. Jung, and Y. Oh, "Improvement of date rate by using equalization in an indoor visible light communication system," in 2008 4th IEEE International Conference on Circuits and Systems for Communications, May 2008, pp. 678-682.

[44] T. Komine and M. Nakagawa, "A study of shadowing on indoor visiblelight wireless communication utilizing plural white LED lightings," in 1st International Symposium on Wireless Communication Systems, Sept 2004, pp. 36-40. 
[45] H. Farahneh, C. Mekhiel, A. Khalifeh, W. Farjow, and X. Fernando, "Shadowing effects on visible light communication channels," in 2016 IEEE Canadian Conference on Electrical and Computer Engineering (CCECE), May 2016, pp. 1-5.

[46] P. Chvojka, S. Zvanovec, P. A. Haigh, and Z. Ghassemlooy, "Channel characteristics of visible light communications within dynamic indoor environment," Journal of Lightwave Technology, vol. 33, no. 9, pp. 1719-1725, May 2015.

[47] C. L. Bas, S. Sahuguede, A. Julien-Vergonjanne, A. Behlouli, P. Combeau, and L. Aveneau, "Human body impact on mobile visible light communication link," in 2016 10th International Symposium on Communication Systems, Networks and Digital Signal Processing (CSNDSP), July 2016, pp. 1-6.

[48] S. Rajagopal, R. D. Roberts, and S. K. Lim, "IEEE 802.15.7 visible light communication: modulation schemes and dimming support," IEEE Communications Magazine, vol. 50, no. 3, pp. 72-82, Mar. 2012.

[49] S. Zhang, S. Watson, J. J. D. McKendry, D. Massoubre, A. Cogman, E. Gu, R. K. Henderson, A. E. Kelly, and M. D. Dawson, "1.5 Gbit/s multi-channel visible light communications using CMOS-controlled GaN-based LEDs," Journal of Lightwave Technology, vol. 31, no. 8, pp. 1211-1216, Apr. 2013.

[50] H. Elgala, R. Mesleh, and H. Haas, "Indoor optical wireless communication: potential and state-of-the-art," IEEE Communications Magazine, vol. 49, no. 9, pp. 56-62, Sept. 2011.

[51] M. Noshad and M. Brandt-Pearce, "Application of expurgated PPM to indoor visible light communications-part I: Single-user systems," Journal of Lightwave Technology, vol. 32, no. 5, pp. 875-882, Mar. 2014

[52] - "Application of expurgated PPM to indoor visible light communications-part II: Access networks," Journal of Lightwave Technology, vol. 32, no. 5, pp. 883-890, Mar. 2014.

[53] D.-S. Shiu and J. M. Kahn, "Differential pulse-position modulation for power-efficient optical communication," IEEE Transactions on Communications, vol. 47, no. 8, pp. 1201-1210, Aug. 1999.

[54] S. H. Lee, K. I. Ahn, and J. K. Kwon, "Multilevel transmission in dimmable visible light communication systems," Journal of Lightwave Technology, vol. 31, no. 20, pp. 3267-3276, Oct. 2013.

[55] R. Singh, T. O'Farrell, and J. P. R. David, "Performance evaluation of IEEE 802.15.7 CSK physical layer," in 2013 IEEE Globecom Workshops, Dec. 2013, pp. 1064-1069.

[56] R. Singh, T. OFarrell, and J. P. R. David, "An enhanced color shift keying modulation scheme for high-speed wireless visible light communications," Journal of Lightwave Technology, vol. 32, no. 14, pp. 2582-2592, July 2014.

[57] J. Jiang, R. Zhang, and L. Hanzo, "Analysis and design of threestage concatenated color-shift keying," IEEE Transactions on Vehicular Technology, vol. 64, no. 11, pp. 5126-5136, Nov. 2015.

[58] Z. Babar, C. Zhu, H. Nguyen, P. Botsinis, D. Alanis, D. Chandra, S. Ng, and L. Hanzo, "Reduced-complexity iterative receiver for improving the IEEE 802.15.7 convolutional-coded color shift keying mode," IEEE Communications Letters, vol. PP, no. 99, pp. 1-1, May 2017.

[59] P. Das, B.-Y. Kim, Y. Park, and K.-D. Kim, "Color-independent VLC based on a color space without sending target color information," Optics Communications, vol. 286, pp. 69-73, Jan. 2013.

[60] P. Das, Y. Park, and K.-D. Kim, "Performance analysis of colorindependent visible light communication using a color-space-based constellation diagram and modulation scheme," Wireless personal communications, vol. 74, no. 2, pp. 665-682, Jan. 2014.

[61] X. Li, R. Mardling, and J. Armstrong, "Channel capacity of IM/DD optical communication systems and of ACO-OFDM," in IEEE ICC 2007, June 2007, pp. 2128-2133.

[62] J. Armstrong and B. Schmidt, "Comparison of asymmetrically clipped optical OFDM and DC-biased optical OFDM in AWGN," Communications Letters, IEEE, vol. 12, no. 5, pp. 343-345, May 2008

[63] D. Tsonev, S. Sinanovic, and H. Haas, "Novel unipolar orthogonal frequency division multiplexing (U-OFDM) for optical wireless," in 2012 IEEE 75th Vehicular Technology Conference (VTC Spring), May 2012, pp. 1-5.

[64] H. Zhang, L. L. Yang, and L. Hanzo, "Compressed sensing improves the performance of subcarrier index-modulation-assisted ofdm," IEEE Access, vol. 4, pp. 7859-7873, Oct. 2016.

[65] — - "Piecewise companding transform assisted optical-OFDM systems for indoor visible light communications," IEEE Access, vol. 5, pp. 295-311, Dec. 2017

[66] "IEEE standard for local and metropolitan area networks-part 15.7: Short-range wireless optical communication using visible light," IEEE Std 802.15.7-2011, pp. 1-309, Sept 2011.
[67] F. Yang, J. Gao, and S. Liu, "Novel visible light communication approach based on hybrid OOK and ACO-OFDM," IEEE Photonics Technology Letters, vol. 28, no. 14, pp. 1585-1588, July 2016.

[68] Y. Yang, Z. Zeng, J. Cheng, and C. Guo, "An enhanced DCO-OFDM scheme for dimming control in visible light communication systems," IEEE Photonics Journal, vol. 8, no. 3, pp. 1-13, June 2016.

[69] W. Huang, J. Takayanagi, T. Sakanaka, and M. Nakagawa, "Atmospheric optical communication system using subcarrier PSK modulation," IEICE Transactions on Communications, vol. 76, no. 9, pp. 1169-1177, Sept. 1993.

[70] J. Armstrong and A. J. Lowery, "Power efficient optical OFDM," Electronics Letters, vol. 42, no. 6, pp. 370-372, Mar. 2006.

[71] S. Dissanayake and J. Armstrong, "Comparison of ACO-OFDM, DCOOFDM and ADO-OFDM in IM/DD systems," Journal of Lightwave Technology, vol. 31, no. 7, pp. 1063-1072, Apr. 2013.

[72] K. Lee and H. Park, "Modulations for visible light communications with dimming control," IEEE Photonics Technology Letters, vol. 23, no. 16, pp. 1136-1138, Aug. 2011.

[73] J. H. Yoo, B. W. Kim, and S. Y. Jung, "Modelling and analysis of M-ary variable pulse position modulation for visible light communications," IET Optoelectronics, vol. 9, no. 5, pp. 184-190, Oct. 2015.

[74] G. Ntogari, T. Kamalakis, J. Walewski, and T. Sphicopoulos, "Combining illumination dimming based on pulse-width modulation with visible-light communications based on discrete multitone," IEEE/OSA Journal of Optical Communications and Networking, vol. 3, no. 1, pp. 56-65, Jan. 2011.

[75] H. Haas, "High-speed wireless networking using visible light," SPIE Newsroom, vol. 10, no. 2.1201304, Apr. 2013.

[76] X. Li, R. Zhang, and L. Hanzo, "Cooperative load balancing in hybrid visible light communications and WiFi," IEEE Transactions on Communications, vol. 63, no. 4, pp. 1319-1329, Apr. 2015.

[77] Y. Wang and H. Haas, "Dynamic load balancing with handover in hybrid Li-Fi and Wi-Fi networks," Journal of Lightwave Technology, vol. 33, no. 22, pp. 4671-4682, Nov. 2015

[78] Z. Wang, C. Yu, W.-D. Zhong, J. Chen, and W. Chen, "Performance of a novel LED lamp arrangement to reduce SNR fluctuation for multiuser visible light communication systems," Optics Express, vol. 20, no. 4, pp. 4564-4573, Feb. 2012

[79] I. Stefan and H. Haas, "Analysis of optimal placement of LED arrays for visible light communication," in 2013 IEEE 77th Vehicular Technology Conference (VTC Spring). IEEE, June 2013, pp. 1-5.

[80] R. Guan, J. Y. Wang, Y.-P. Wen, J.-B. Wang, and M. Chen, "Psobased led deployment optimization for visible light communications," in 2013 International Conference on Wireless Communications and Signal Processing, Oct. 2013, pp. 1-6.

[81] G. W. Marsh and J. M. Kahn, "Channel reuse strategies for indoor infrared wireless communications," IEEE Transactions on Communications, vol. 45, no. 10, pp. 1280-1290, Oct. 1997.

[82] B. Ghimire and H. Haas, "Self-organising interference coordination in optical wireless networks," EURASIP Journal on Wireless Communications and Networking, vol. 2012, no. 1, pp. 1-15, Dec. 2012.

[83] J.-Y. Sung, W.-F. Lin, Y.-F. Wu, C.-W. Chow, and C.-H. Yeh, "Design of visible light communication system for maintaining uniform data rate," in Novel Optical Systems Design and Optimization XVII, vol. 9193, Jan. 2014

[84] C. Chen, S. Videv, D. Tsonev, and H. Haas, "Fractional frequency reuse in DCO-OFDM-based optical attocell networks," Journal of Lightwave Technology, vol. 33, no. 19, pp. 3986-4000, Oct. 2015.

[85] G. B. Prince and T. D. C. Little, "On the performance gains of cooperative transmission concepts in intensity modulated direct detection visible light communication networks," in 2010 6th International Conference on Wireless and Mobile Communications (ICWMC), Sept 2010, pp. 297-302.

[86] Y. Wu, A. Yang, L. Feng, L. Zuo, and Y. nan Sun, "Modulation based cells distribution for visible light communication," Optics Express, vol. 20, no. 22, pp. 24 196-24 208, Oct. 2012.

[87] M. S. Alouini and A. J. Goldsmith, "Area spectral efficiency of cellular mobile radio systems," IEEE Transactions on Vehicular Technology, vol. 48, no. 4, pp. 1047-1066, July 1999.

[88] C. Chen, D. Tsonev, and H. Haas, "Joint transmission in indoor visible light communication downlink cellular networks," in IEEE GLOBECOM Workshops 2013, Dec. 2013, pp. 1127-1132.

[89] X. Li, F. Jin, R. Zhang, J. Wang, Z. Xu, and L. Hanzo, "Users first: User-centric cluster formation for interference-mitigation in visiblelight networks," IEEE Transactions on Wireless Communications, vol. 15 , no. 1, pp. 39-53, Jan. 2016. 
[90] R. Zhang, H. Claussen, H. Haas, and L. Hanzo, "Energy efficient visible light communications relying on amorphous cells," IEEE Journal on Selected Areas in Communications, vol. 34, no. 4, pp. 894-906, Apr. 2016.

[91] X. Li, Y. Huo, R. Zhang, and L. Hanzo, "User-centric visible light communications for energy-efficient scalable video streaming," IEEE Transactions on Green Communications and Networking, vol. 1, no. 1, pp. 59-73, Mar. 2017.

[92] C. Chen, D. A. Basnayaka, and H. Haas, "Downlink performance of optical attocell networks," Journal of Lightwave Technology, vol. 34, no. 1, pp. 137-156, Jan. 2016.

[93] J. G. Andrews, F. Baccelli, and R. K. Ganti, "A tractable approach to coverage and rate in cellular networks," IEEE Transactions on Communications, vol. 59, no. 11, pp. 3122-3134, Nov. 2011.

[94] Y. Tao, X. Liang, J. Wang, and C. Zhao, "Scheduling for indoor visible light communication based on graph theory," Optics Express, vol. 23, no. 3, pp. 2737-2752, Feb. 2015.

[95] X. Li, R. Zhang, J. Wang, and L. Hanzo, "Cell-centric and user-centric multi-user scheduling in visible light communication aided networks," in 2015 IEEE International Conference on Communications (ICC), June 2015, pp. 5120-5125.

[96] S. Feng, X. Li, R. Zhang, M. Jiang, and L. Hanzo, "Hybrid positioning aided amorphous-cell assisted user-centric visible light downlink techniques," IEEE Access, vol. 4, pp. 2705-2713, May 2016.

[97] B. Dai and W. Yu, "Sparse beamforming and user-centric clustering for downlink cloud radio access network," IEEE Access, vol. 2, pp. 1326-1339, Oct. 2014.

[98] T. R. Lakshmana, A. Tlli, R. Devassy, and T. Svensson, "Precoder design with incomplete feedback for joint transmission," IEEE Transactions on Wireless Communications, vol. 15, no. 3, pp. 1923-1936, Mar. 2016.

[99] Z. Chen, X. Hou, and C. Yang, "Training resource allocation for user-centric base station cooperation networks," IEEE Transactions on Vehicular Technology, vol. 65, no. 4, pp. 2729-2735, Apr. 2016.

[100] D. Liu, S. Han, C. Yang, and Q. Zhang, "Semi-dynamic user-specific clustering for downlink cloud radio access network," IEEE Transactions on Vehicular Technology, vol. 65, no. 4, pp. 2063-2077, Apr. 2016.

[101] C. Pan, H. Zhu, N. J. Gomes, and J. Wang, "Joint user selection and energy minimization for ultra-dense multi-channel C-RAN with incomplete CSI," IEEE Journal on Selected Areas in Communications, vol. 35, no. 8, pp. 1809-1824, Aug. 2017.

[102] J. W. J. Y. Cunhua Pan, Maged Elkashlan and L. Hanzo, "Usercentric C-RAN architecture for ultra-dense 5G networks: Challenges and methodologies," IEEE Wireless Communications (accepted), 2017.

[103] M. Biagi, S. Pergoloni, and A. M. Vegni, "LAST: A framework to localize, access, schedule, and transmit in indoor VLC systems," Journal of Lightwave Technology, vol. 33, no. 9, pp. 1872-1887, May 2015

[104] S. G. Wilson, M. Brandt-Pearce, Q. Cao, and J. H. Leveque, "Freespace optical MIMO transmission with Q-ary PPM," IEEE Transactions on Communications, vol. 53, no. 8, pp. 1402-1412, Aug. 2005.

[105] T. A. Tsiftsis, H. G. Sandalidis, G. K. Karagiannidis, and M. Uysal, "Optical wireless links with spatial diversity over strong atmospheric turbulence channels," IEEE Transactions on Wireless Communications, vol. 8, no. 2, pp. 951-957, Feb. 2009.

[106] L. Zeng, D. C. O’Brien, H. L. Minh, G. E. Faulkner, K. Lee, D. Jung, Y. Oh, and E. T. Won, "High data rate multiple input multiple output (MIMO) optical wireless communications using white LED lighting," IEEE Journal on Selected Areas in Communications, vol. 27, no. 9, pp. 1654-1662, Dec. 2009.

[107] K. D. Dambul, D. C. O'Brien, and G. Faulkner, "Indoor optical wireless MIMO system with an imaging receiver," IEEE Photonics Technology Letters, vol. 23, no. 2, pp. 97-99, Jan. 2011.

[108] T. Q. Wang, Y. A. Sekercioglu, and J. Armstrong, "Analysis of an optical wireless receiver using a hemispherical lens with application in MIMO visible light communications," Journal of Lightwave Technology, vol. 31, no. 11, pp. 1744-1754, June 2013.

[109] A. H. Azhar, T. A. Tran, and D. O'Brien, "Demonstration of high-speed data transmission using MIMO-OFDM visible light communications," in 2010 IEEE Globecom Workshops, Dec. 2010, pp. 1052-1056.

[110] A. H. Azhar, T. Tran, and D. O'Brien, "A gigabit/s indoor wireless transmission using MIMO-OFDM visible-light communications," IEEE Photonics Technology Letters, vol. 25, no. 2, pp. 171-174, Jan. 2013.

[111] Z. Yu, R. J. Baxley, and G. T. Zhou, "Multi-user MISO broadcasting for indoor visible light communication," in 2013 IEEE International
Conference on Acoustics, Speech and Signal Processing (ICASSP), May 2013, pp. 4849-4853.

[112] B. Li, J. Wang, R. Zhang, H. Shen, C. Zhao, and L. Hanzo, "Multiuser MISO transceiver design for indoor downlink visible light communication under per-LED optical power constraints," IEEE Photonics Journal, vol. 7, no. 4, pp. 1-15, Aug. 2015.

[113] M. Biagi, A. M. Vegni, and T. D. C. Little, "LAT indoor MIMO-VLC - localize, access and transmit," in 2012 International Workshop on Optical Wireless Communications (IWOW), Oct. 2012, pp. 1-3.

[114] Q. Wang, Z. Wang, and L. Dai, "Multiuser MIMO-OFDM for visible light communications," IEEE Photonics Journal, vol. 7, no. 6, pp. 111, Dec. 2015

[115] H. Shen, Y. Deng, W. Xu, and C. Zhao, "Rate-maximized zero-forcing beamforming for VLC multiuser MISO downlinks," IEEE Photonics Journal, vol. 8, no. 1, pp. 1-13, Feb. 2016

[116] J. M. Kahn, R. You, P. Djahani, A. G. Weisbin, B. K. Teik, and A. Tang, "Imaging diversity receivers for high-speed infrared wireless communication," IEEE Communications Magazine, vol. 36, no. 12, pp. 88-94, Dec. 1998.

[117] C. J. Chen and L.-C. Wang, "A unified capacity analysis for wireless systems with joint multiuser scheduling and antenna diversity in Nakagami fading channels," IEEE Transactions on Communications, vol. 54, no. 3, pp. 469-478, Mar. 2006.

[118] C. j. Chen and L. c. Wang, "Performance analysis of scheduling in multiuser MIMO systems with zero-forcing receivers," IEEE Journal on Selected Areas in Communications, vol. 25, no. 7, pp. 1435-1445, Sept. 2007.

[119] R. Morsi, D. S. Michalopoulos, and R. Schober, "Multiuser scheduling schemes for simultaneous wireless information and power transfer over fading channels," IEEE Transactions on Wireless Communications, vol. 14, no. 4, pp. 1967-1982, Apr. 2015.

[120] E. Castaneda, A. Silva, R. Samano-Robles, and A. Gameiro, "Distributed linear precoding and user selection in coordinated multicell systems," IEEE Transactions on Vehicular Technology, vol. 65, no. 7, pp. 4887-4899, July 2016.

[121] D. Bykhovsky and S. Arnon, "Multiple access resource allocation in visible light communication systems," Journal of Lightwave Technology, vol. 32, no. 8, pp. 1594-1600, Apr. 2014.

[122] X. Huang, X. Fu, and W. Xu, "Incremental scheduling scheme for indoor visible light communication," Electronics Letters, vol. 51, no. 3, pp. 268-270, Feb. 2015

[123] O. Babatundi, L. Qian, and J. Cheng, "Downlink scheduling in visible light communications," in WCSP 2014, Oct. 2014, pp. 1-6.

[124] W. C. Kim, C. S. Bae, S. Y. Jeon, S. Y. Pyun, and D. H. Cho, "Efficient resource allocation for rapid link recovery and visibility in visible-light local area networks," IEEE Transactions on Consumer Electronics, vol. 56, no. 2, pp. 524-531, May 2010.

[125] K. H. Park, Y. C. Ko, and M. S. Alouini, "On the power and offset allocation for rate adaptation of spatial multiplexing in optical wireless MIMO channels," IEEE Transactions on Communications, vol. 61, no. 4, pp. 1535-1543, Apr. 2013.

[126] H. Marshoud, V. M. Kapinas, G. K. Karagiannidis, and S. Muhaidat, "Non-orthogonal multiple access for visible light communications," IEEE Photonics Technology Letters, vol. 28, no. 1, pp. 51-54, Jan. 2016

[127] R. Jiang, Z. Wang, Q. Wang, and L. Dai, "Multi-user sum-rate optimization for visible light communications with lighting constraints," Journal of Lightwave Technology, vol. 34, no. 16, pp. 3943-3952, Aug. 2016.

[128] C. E. Shannon, "A mathematical theory of communication," ACM SIGMOBILE Mobile Computing and Communications Review, vol. 5, no. 1, pp. 3-55, Jan. 2001. [Online]. Available: http://doi.acm.org/10.1145/584091.584093

[129] J. Andrews, S. Singh, Q. Ye, X. Lin, and H. Dhillon, "An overview of load balancing in hetnets: old myths and open problems," IEEE Wireless Communications, vol. 21, no. 2, pp. 18-25, Apr. 2014.

[130] T. Bu, L. Li, and R. Ramjee, "Generalized proportional fair scheduling in third generation wireless data networks," in Proceedings of INFO COM 2006, Apr. 2006, pp. 1-12.

[131] L. Li, M. Pal, and Y. Yang, "Proportional fairness in multi-rate wireless LANs," in Proceedings of INFOCOM 2008, Apr. 2008, pp. 1-9.

[132] K. Son, S. Chong, and G. Veciana, "Dynamic association for load balancing and interference avoidance in multi-cell networks," IEEE Transactions on Wireless Communications, vol. 8, no. 7, pp. 35663576 , July 2009

[133] H. Burchardt, S. Sinanovic, Z. Bharucha, and H. Haas, "Distributed and autonomous resource and power allocation for wireless networks," 
IEEE Transactions on Communications, vol. 61, no. 7, pp. 2758-2771, July 2013.

[134] F. Heliot, M. Imran, and R. Tafazolli, "Low-complexity energy-efficient resource allocation for the downlink of cellular systems," IEEE Transactions on Communications, vol. 61, no. 6, pp. 2271-2281, June 2013.

[135] M. Ismail and W. Zhuang, "A distributed multi-service resource allocation algorithm in heterogeneous wireless access medium," IEEE Journal on Selected Areas in Communications, vol. 30, no. 2, pp. 425432, Feb. 2012.

[136] Q. Ye, B. Rong, Y. Chen, M. Al-Shalash, C. Caramanis, and J. Andrews, "User association for load balancing in heterogeneous cellular networks," IEEE Transactions on Wireless Communications, vol. 12, no. 6, pp. 2706-2716, June 2013.

[137] D. Palomar and M. Chiang, "A tutorial on decomposition methods for network utility maximization," IEEE Journal on Selected Areas in Communications, vol. 24, no. 8, pp. 1439-1451, Aug. 2006

[138] D. P. Bertsekas, "Nonlinear programming," 1999.

[139] S. P. Boyd and L. Vandenberghe, Convex optimization. Cambridge University Press, 2004.

[140] I. Stefan and H. Haas, "Hybrid visible light and radio frequency communication systems," in 2014 IEEE 80th Vehicular Technology Conference (VTC2014-Fall), Sept 2014, pp. 1-5.

[141] F. Jin, R. Zhang, and L. Hanzo, "Resource allocation under delayguarantee constraints for heterogeneous visible-light and RF femtocell," IEEE Transactions on Wireless Communications, vol. 14, no. 2, pp. 1020-1034, Feb. 2015

[142] X. Wu, M. Safari, and H. Haas, "Bidirectional allocation game in visible light communications," in 2016 IEEE 83rd Vehicular Technology Conference (VTC Spring), May 2016, pp. 1-5.

[143] L. Li, Y. Zhang, B. Fan, and H. Tian, "Mobility-aware load balancing scheme in hybrid VLC-LTE networks," IEEE Communications Letters, vol. 20, no. 11, pp. 2276-2279, Nov. 2016.

[144] M. Kashef, M. Ismail, M. Abdallah, K. A. Qaraqe, and E. Serpedin, "Energy efficient resource allocation for mixed RF/VLC heterogeneous wireless networks," IEEE Journal on Selected Areas in Communications, vol. 34, no. 4, pp. 883-893, Apr. 2016

[145] H. Haas, L. Yin, Y. Wang, and C. Chen, "What is LiFi?" Journal of Lightwave Technology, vol. 34, no. 6, pp. 1533-1544, Mar. 2016.

[146] B. V. Quang, R. V. Prasad, and I. Niemegeers, "A survey on handoffs: Lessons for $60 \mathrm{GHz}$ based wireless systems," IEEE Communications Surveys \& Tutorials, vol. 14, no. 1, pp. 64-86, First Quarter 2012

[147] S. K. Ray, K. Pawlikowski, and H. Sirisena, "Handover in mobile WiMAX networks: The state of art and research issues," IEEE Communications Surveys \& Tutorials, vol. 12, no. 3, pp. 376-399, Third Quarter 2010.

[148] D. Xenakis, N. Passas, L. Merakos, and C. Verikoukis, "Mobility management for femtocells in LTE-Advanced: Key aspects and survey of handover decision algorithms," IEEE Communications Surveys \& Tutorials, vol. 16, no. 1, pp. 64-91, First Quarter 2014.

[149] A. M. Vegni and T. D. C. Little, "Handover in VLC systems with cooperating mobile devices," in 2012 International Conference on Computing, Networking and Communications, Jan. 2012, pp. 126-130.

[150] M. Rahaim, A. Vegni, and T. D. C. Little, "A hybrid radio frequency and broadcast visible light communication system," in IEEE GLOBECOM Workshops 2011, Dec. 2011, pp. 792-796.

[151] S. Liang, H. Tian, B. Fan, and R. Bai, "A novel vertical handover algorithm in a hybrid visible light communication and LTE system," in 2015 IEEE 82nd Vehicular Technology Conference, Sept 2015, pp. $1-5$.

[152] J. Hou and D. C. O'Brien, "Vertical handover-decision-making algorithm using fuzzy logic for the integrated radio-and-OW system," IEEE Transactions on Wireless Communications, vol. 5, no. 1, pp. 176-185, Jan. 2006.

[153] F. Wang, Z. Wang, C. Qian, L. Dai, and Z. Yang, "Efficient vertical handover scheme for heterogeneous VLC-RF systems," IEEE/OSA Journal of Optical Communications and Networking, vol. 7, no. 12, pp. 1172-1180, Dec. 2015.

[154] L. Hanzo, P. J. Cherriman, and J. Streit, Wireless video communications: second to third generation and beyond. IEEE Press-John Wiley \& Sons, 2001

[155] Y. Huo, C. Hellge, T. Wiegand, and L. Hanzo, "A tutorial and review on inter-layer FEC coded layered video streaming," IEEE Communications Surveys \& Tutorials, vol. 17, no. 2, pp. 1166-1207, Second Quarter 2015.

[156] J. Jiang, Y. Huo, F. Jin, P. Zhang, Z. Wang, Z. Xu, H. Haas, and L. Hanzo, "Video streaming in the multiuser indoor visible light downlink," IEEE Access, vol. 3, pp. 2959-2986, Dec. 2015.
[157] L. Hanzo, P. Cherriman, and J. Streit, Video compression and communications: from basics to $H .261, H .263, H .264$, MPEG4 for DVB and HSDPA-style adaptive turbo-transceivers. John Wiley \& Sons, 2007.

[158] G. Sullivan, J. Ohm, W.-J. Han, and T. Wiegand, "Overview of the high efficiency video coding (HEVC) standard," IEEE Transactions on Circuits and Systems for Video Technology, vol. 22, no. 12, pp. 16491668, Dec. 2012.

[159] J. Boyce, Y. Ye, J. Chen, and A. Ramasubramonian, "Overview of SHVC: Scalable extensions of the high efficiency video coding (HEVC) standard," IEEE Transactions on Circuits and Systems for Video Technology, vol. PP, no. 99, pp. 1-1, July 2015.

[160] Y. Wang and Q.-F. Zhu, "Error control and concealment for video communication: a review," Proceedings of the IEEE, vol. 86, no. 5, pp. 974-997, May 1998.

[161] Y. Huo, M. El-Hajjar, and L. Hanzo, "Inter-layer FEC aided unequal error protection for multilayer video transmission in mobile TV," IEEE Transactions on Circuits and Systems for Video Technology, vol. 23, no. 9, pp. 1622-1634, Sept 2013.

[162] P. Pahalawatta, R. Berry, T. Pappas, and A. Katsaggelos, "Contentaware resource allocation and packet scheduling for video transmission over wireless networks," IEEE Journal on Selected Areas in Communications, vol. 25, no. 4, pp. 749-759, May 2007.

[163] Y. Li, Z. Li, M. Chiang, and A. R. Calderbank, "Content-aware distortion-fair video streaming in congested networks," IEEE Transactions on Multimedia, vol. 11, no. 6, pp. 1182-1193, Oct. 2009.

[164] M. Ismail, W. Zhuang, and S. Elhedhli, "Energy and content aware multi-homing video transmission in heterogeneous networks," IEEE Transactions on Wireless Communications, vol. 12, no. 7, pp. 36003610, July 2013.

[165] J. Vucic, C. Kottke, L. Fernandez, S. Nerreter, J. Walewski, K. Habel, and K. D. Langer, "Video broadcast via a lamp," in 2010 12th International Conference on Transparent Optical Networks, June 2010 , pp. $1-4$.

[166] J. Rufo, J. Rabadan, F. Delgado, C. Quintana, and R. Perez-Jimenez, "Experimental evaluation of video transmission through LED illumination devices," IEEE Transactions on Consumer Electronics, vol. 56 , no. 3, pp. 1411-1416, Aug. 2010

[167] W. Chen, C. Yu, Y. S. Kwok, and F. Chin, "Video transmission system based on visible light communication," in 2011 International Conference on Information Photonics and Optical Communications (IPOC), Oct. 2011, pp. 1-3

[168] L. C. Png, "A fully integrated audio, video, and data VLC transceiver system for smartphones and tablets," in 2013 IEEE International Symposium on Consumer Electronics (ISCE), June 2013, pp. 249-250.

[169] Y. Huo, T. Wang, R. Maunder, and L. Hanzo, "Motion-aware meshstructured trellis for correlation modelling aided distributed multi-view video coding," IEEE Transactions on Image Processing, vol. 23, no. 1, pp. 319-331, Jan. 2014.

[170] D. Vukobratovic, V. Stankovic, D. Sejdinovic, L. Stankovic, an Z. Xiong, "Scalable video multicast using expanding window fountain codes," IEEE Transactions on Multimedia, vol. 11, no. 6, pp. 10941104, Oct. 2009.

[171] P. Li, H. Zhang, B. Zhao, and S. Rangarajan, "Scalable video multicast with adaptive modulation and coding in broadband wireless data systems," IEEE/ACM Transactions on Networking, vol. 20, no. 1, pp. 57-68, Feb. 2012

[172] H. Zhou, Y. Ji, X. Wang, and B. Zhao, "Joint resource allocation and user association for SVC multicast over heterogeneous cellula networks," IEEE Transactions on Wireless Communications, vol. 14 no. 7, pp. 3673-3684, July 2015.

[173] H. Schwarz, D. Marpe, and T. Wiegand, "Overview of the scalable video coding extension of the H.264/AVC standard," IEEE Transactions on Circuits and Systems for Video Technology, vol. 17, no. 9, pp. 1103-1120, Sept 2007.

[174] C. Singhal, S. De, R. Trestian, and G.-M. Muntean, "Joint optimization of user-experience and energy-efficiency in wireless multimedia broadcast," IEEE Transactions on Mobile Computing, vol. 13, no. 7, pp. 1522-1535, July 2014.

[175] M. Ismail, W. Zhuang, and S. Elhedhli, "Energy and content aware multi-homing video transmission in heterogeneous networks," IEEE Transactions on Wireless Communications, vol. 12, no. 7, pp. 36003610, July 2013.

[176] S.-P. Chuah, Z. Chen, and Y.-P. Tan, "Energy-efficient resource allocation and scheduling for multicast of scalable video over wireless networks," IEEE Transactions on Multimedia, vol. 14, no. 4, pp. 1324 1336, Aug. 2012. 
[177] C. Zhu, Y. Huo, J. Jiang, H. Sun, C. Dong, R. Zhang, and L. Hanzo, "Hierarchical colour-shift-keying aided layered video streaming for the visible light downlink," IEEE Access, vol. 4, pp. 3127-3152, June 2016.

[178] H. Wymeersch, J. Lien, and M. Z. Win, "Cooperative localization in wireless networks," Proceedings of the IEEE, vol. 97, no. 2, pp. 427450, Feb. 2009.

[179] K. Chintalapudi, A. Padmanabha Iyer, and V. N. Padmanabhan, "Indoor localization without the pain," in Proceedings of the Sixteenth Annual International Conference on Mobile Computing and Networking, ser. MobiCom '10. New York, NY, USA: ACM, Sept. 2010, pp. 173-184. [Online]. Available: http://doi.acm.org/10.1145/1859995.1860016

[180] L. Li, P. Hu, C. Peng, G. Shen, and F. Zhao, "Epsilon: A visible light based positioning system," in 11th USENIX Symposium on Networked Systems Design and Implementation, Apr. 2014, pp. 331-343.

[181] S. Y. Jung, S. Hann, and C. S. Park, "TDOA-based optical wireless indoor localization using LED ceiling lamps," IEEE Transactions on Consumer Electronics, vol. 57, no. 4, pp. 1592-1597, Nov. 2011.

[182] H. S. Kim, D. R. Kim, S. H. Yang, Y. H. Son, and S. K. Han, "An indoor visible light communication positioning system using a RF carrier allocation technique," Journal of Lightwave Technology, vol. 31, no. 1, pp. 134-144, Jan. 2013.

[183] J. Armstrong, Y. A. Sekercioglu, and A. Neild, "Visible light positioning: a roadmap for international standardization," IEEE Communications Magazine, vol. 51, no. 12, pp. 68-73, Dec. 2013.

[184] S. H. Yang, H. S. Kim, Y. H. Son, and S. K. Han, "Three-dimensional visible light indoor localization using AOA and RSS with multiple optical receivers," Journal of Lightwave Technology, vol. 32, no. 14, pp. 2480-2485, July 2014.

[185] Y. Hou, S. Xiao, H. Zheng, and W. Hu, "Multiple access scheme based on block encoding time division multiplexing in an indoor positioning system using visible light," IEEE/OSA Journal of Optical Communications and Networking, vol. 7, no. 5, pp. 489-495, May 2015.

[186] M. Yasir, S. W. Ho, and B. N. Vellambi, "Indoor position tracking using multiple optical receivers," Journal of Lightwave Technology, vol. 34, no. 4, pp. 1166-1176, Feb. 2016.

[187] W. Xu, J. Wang, H. Shen, H. Zhang, and X. You, "Indoor positioning for multiphotodiode device using visible-light communications," IEEE Photonics Journal, vol. 8, no. 1, pp. 1-11, Feb. 2016.

[188] H. S. Liu and G. Pang, "Positioning beacon system using digital camera and LEDs," IEEE Transactions on Vehicular Technology, vol. 52, no. 2, pp. 406-419, Mar. 2003.

[189] C. Li and S. Shimamoto, "An open traffic light control model for reducing vehicles $\mathrm{CO}_{2}$ emissions based on ETC vehicles," IEEE Transactions on Vehicular Technology, vol. 61, no. 1, pp. 97-110, Jan. 2012.

[190] I. Takai, S. Ito, K. Yasutomi, K. Kagawa, M. Andoh, and S. Kawahito, "LED and CMOS image sensor based optical wireless communication system for automotive applications," IEEE Photonics Journal, vol. 5, no. 5, pp. $6801418-6801418$, Oct. 2013.

[191] S. H. Yu, O. Shih, H. M. Tsai, N. Wisitpongphan, and R. D. Roberts, "Smart automotive lighting for vehicle safety," IEEE Communications Magazine, vol. 51, no. 12, pp. 50-59, Dec. 2013.

[192] T. Yamazato, I. Takai, H. Okada, T. Fujii, T. Yendo, S. Arai, M. Andoh, T. Harada, K. Yasutomi, K. Kagawa, and S. Kawahito, "Image-sensorbased visible light communication for automotive applications," IEEE Communications Magazine, vol. 52, no. 7, pp. 88-97, July 2014.

[193] T. Yamazato, M. Kinoshita, S. Arai, E. Souke, T. Yendo, T. Fujii, K. Kamakura, and H. Okada, "Vehicle motion and pixel illumination modeling for image sensor based visible light communication," IEEE Journal on Selected Areas in Communications, vol. 33, no. 9, pp. 1793 1805, Sept 2015

[194] M. Uysal, Z. Ghassemlooy, A. Bekkali, A. Kadri, and H. Menouar, "Visible light communication for vehicular networking: Performance study of a V2V system using a measured headlamp beam pattern model," IEEE Vehicular Technology Magazine, vol. 10, no. 4, pp. 4553, Dec. 2015

[195] B. Zhang, K. Ren, G. Xing, X. Fu, and C. Wang, "SBVLC: Secure barcode-based visible light communication for smartphones," IEEE Transactions on Mobile Computing, vol. 15, no. 2, pp. 432-446, Feb. 2016.

[196] S. Hranilovic, L. Lampe, and S. Hosur, "Visible light communications: the road to standardization and commercialization (part 1) [guest editorial]," IEEE Communications Magazine, vol. 51, no. 12, pp. 24 25, Dec. 2013

[197] "St. cloud first to sign on for new technology," Nov. 2010. [Online]. Available: http://www.tmenet.com/usubmit/2010/11/19/5148608.htm.
[198] “Wireless data from every light bulb," July 2011. [Online]. Available: https://www.ted.com/talks/harald_haas_wireless_data_from_every_light_bulb?

[199] "Where are the discounts? Carrefours LED supermarket lighting from philips will guide you," May 2015. [Online]. Available: http://www.philips.com/aw/about/news/archive/standard/news/press/2015/20150521-Whereare-the-discounts-Carrefours-LED-supermarket-lighting-from-Philipswill-guide-you.html\#.VgAF-t-qpBc.

[200] A. Jovicic, J. Li, and T. Richardson, "Visible light communication: opportunities, challenges and the path to market," IEEE Communications Magazine, vol. 51, no. 12, pp. 26-32, Dec. 2013.

[201] H. Zhang, L. L. Yang, and L. Hanzo, "Compressed impairment sensingassisted and interleaved-double-fft-aided modulation improves broadband power line communications subjected to asynchronous impulsive noise," IEEE Access, vol. 4, pp. 81-96, Dec. 2016.

[202] — - "Performance analysis of orthogonal frequency division multiplexing systems in dispersive indoor power line channels inflicting asynchronous impulsive noise," IET Communications, vol. 10, no. 5, pp. 453-461, Apr. 2016.

[203] T. Komine and M. Nakagawa, "Integrated system of white LED visible-light communication and power-line communication," IEEE Transactions on Consumer Electronics, vol. 49, no. 1, pp. 71-79, Feb. 2003.

[204] T. Komine, S. Haruyama, and M. Nakagawa, "Performance evaluation of narrowband ofdm on integrated system of power line communication and visible light wireless communication," in 2006 1st International Symposium on Wireless Pervasive Computing, Jan. 2006, pp. 1-6.

[205] C. H. Lo and N. Ansari, "The progressive smart grid system from both power and communications aspects," IEEE Communications Surveys \& Tutorials, vol. 14, no. 3, pp. 799-821, Third Quarter 2012.

[206] M. Kavehrad and P. Amirshahi, "Hybrid MV-LV power lines and white light emitting diodes for triple-play broadband access communications," IEC Comprehensive Report on Achieving the Triple Play: Technologies and Business Models for Success, pp. 167-178, 2006.

[207] H. Ma, L. Lampe, and S. Hranilovic, "Integration of indoor visible light and power line communication systems," in 2013 IEEE 17th International Symposium on Power Line Communications and Its Applications, Mar. 2013, pp. 291-296.

[208] J. Song, W. Ding, F. Yang, H. Yang, B. Yu, and H. Zhang, "An indoor broadband broadcasting system based on PLC and VLC," IEEE Transactions on Broadcasting, vol. 61, no. 2, pp. 299-308, June 2015.

[209] A. D. Familua, A. R. Ndjiongue, K. Ogunyanda, L. Cheng, H. C. Ferreira, and T. G. Swart, "A semi-hidden markov modeling of a low complexity FSK-OOK in-house PLC and VLC integration," in 2015 IEEE International Symposium on Power Line Communications and Its Applications (ISPLC), Mar. 2015, pp. 199-204.

[210] Y. Yan, W. Ding, H. Yang, and J. Song, "The video transmission platform for the PLC and VLC integrated system," in 2015 IEEE International Symposium on Broadband Multimedia Systems and Broadcasting, June 2015, pp. 1-5.

[211] X. Ma, J. Gao, F. Yang, W. Ding, H. Yang, and J. Song, "Integrated power line and visible light communication system compatible with multi-service transmission," IET Communications, vol. 11, no. 1, pp. 104-111, 2017.

[212] T. Nguyen, M. Z. Chowdhury, and Y. M. Jang, "A novel link switching scheme using pre-scanning and RSS prediction in visible light communication networks," EURASIP Journal on Wireless Communications and Networking, vol. 2013, no. 1, pp. 1-17, Dec. 2013.

[213] Y. H. Kim, W. A. Cahyadi, and Y. H. Chung, "Experimental demonstration of vlc-based vehicle-to-vehicle communications under fog conditions," IEEE Photonics Journal, vol. 7, no. 6, pp. 1-9, Dec. 2015.

[214] H. Farahneh, A. Khalifeh, and X. Fernando, "An outdoor multi path channel model for vehicular visible light communication systems," in 2016 Photonics North (PN), May 2016, pp. 1-1.

[215] N. Lourenco, D. Terra, N. Kumar, L. N. Alves, and R. L. Aguiar, "Visible light communication system for outdoor applications," in 2012 8th International Symposium on Communication Systems, Networks Digital Signal Processing (CSNDSP), July 2012, pp. 1-6.

[216] Y. J. Zhu, Z. G. Sun, J. K. Zhang, and Y. Y. Zhang, "A fast blind detection algorithm for outdoor visible light communications," IEEE Photonics Journal, vol. 7, no. 6, pp. 1-8, Dec. 2015.

[217] D. Q. Hien and M. Yoo, "Handover in outdoor visible light communication system," in 2017 International Conference on Information Networking (ICOIN), Jan. 2017, pp. 67-69.

[218] R. Mesleh, H. Elgala, and H. Haas, "LED nonlinearity mitigation techniques in optical wireless OFDM communication systems," Journal 
of Optical Communications and Networking, vol. 4, no. 11, pp. 865875, Oct. 2012.

[219] Z. Wang, Q. Wang, S. Chen, and L. Hanzo, "An adaptive scaling and biasing scheme for OFDM-based visible light communication systems," Optics express, vol. 22, no. 10, pp. 12 707-12 715, May 2014.

[220] H. Elgala, R. Mesleh, and H. Haas, "Non-linearity effects and predistortion in optical OFDM wireless transmission using LEDs," International Journal of Ultra Wideband Communications and Systems, vol. 1, no. 2, pp. 143-150, Oct. 2009.

[221] K. Ying, Z. Yu, R. J. Baxley, H. Qian, G. K. Chang, and G. T. Zhou, "Nonlinear distortion mitigation in visible light communications," IEEE Wireless Communications, vol. 22, no. 2, pp. 36-45, Apr. 2015.

[222] H. Qian, S. Yao, S. Cai, and T. Zhou, "Adaptive postdistortion for nonlinear LEDs in visible light communications," IEEE Photonics Journal, vol. 6, no. 4, pp. 1-8, June 2014.

[223] Y. Wang, L. Tao, X. Huang, J. Shi, and N. Chi, "Enhanced performance of a high-speed WDM CAP64 VLC system employing volterra seriesbased nonlinear equalizer," IEEE Photonics Journal, vol. 7, no. 3, pp. 1-7, May 2015. 